\title{
IMPLICIT-EXPLICIT MULTISTEP FORMULATIONS FOR FINITE ELEMENT DISCRETISATIONS USING CONTINUOUS INTERIOR PENALTY
}

\author{
ERIK BURMAN ${ }^{1}$ AND JOHNNy GUZMÁN ${ }^{2 * *}$
}

\begin{abstract}
We consider a finite element method with symmetric stabilisation for the discretisation of the transient convection-diffusion equation. For the time-discretisation we consider either the second order backwards differentiation formula or the Crank-Nicolson method. Both the convection term and the associated stabilisation are treated explicitly using an extrapolated approximate solution. We prove stability of the method and the $\tau^{2}+h^{p+\frac{1}{2}}$ error estimates for the $L^{2}$-norm under either the standard hyperbolic CFL condition, when piecewise affine $(p=1)$ approximation is used, or in the case of finite element approximation of order $p \geq 1$, a stronger, so-called 4/3-CFL, i.e. $\tau \leq C h^{4 / 3}$. The theory is illustrated with some numerical examples.
\end{abstract}

Mathematics Subject Classification. 65M60.

Received June 7, 2021. Accepted December 14, 2021.

\section{INTRODUCTION}

In the computational solution of convection-diffusion problems it is highly advantageous to treat the convection term explicitly and the diffusion term implicitly. Although there is an important literature on the topic there seems to be very few works that show that implicit-explicit (IMEX) methods are robust under finite element discretisation both in the convection and the diffusion dominated regimes. Indeed most works on IMEX methods with finite element discretisations assume that the diffusion dominates, or at least that the mesh Peclet number, defined by

$$
\mathrm{Pe}:=\frac{|u| h}{\mu}
$$

where $u$ denotes the fluid velocity, $\mu$ the viscosity coefficient and $h$ the local mesh size, is small. A standard assumption is that the product of the Courant number and the Peclet number is small $[1-3,13,24]$. Most other works on IMEX methods typically consider a stability region type analysis that is unsuitable for a quantitative finite element analysis $[4,5,17,18]$, or time-splitting approaches, where the convective terms typically is handled using a robust low order method [14,20,22]. The derivation of a (at least) second order finite element method, which is robust for all Peclet number, without imposing to strict conditions on the discretisation parameters remains a challenging problem. To pass from the analysis of the semi-discrete case to a fully discrete case

Keywords and phrases. IMEX - multistep, continuous interior penalty.

1 Department of Mathematics, University College London, London, UK.

2 Division of Applied Mathematics, Brown University, Providence, RI, USA.

*Corresponding author: johnny_guzman@brown.edu 
typically requires the use of energy methods as advocated in [19]. This was the route taken in [8] where a second order implicit explicit Runge-Kutta method was considered for $H^{1}$-conforming finite element approximations of convection-diffusion equations using interior penalty to guarantee stability in the high Peclet regime and more recently [26] where a local discontinuous Galerkin methods with 2nd and 3rd order Runge-Kutta IMEX schemes are considered. Explicit Runge-Kutta methods have been very successful for the approximation of hyperbolic equations often in combination with discontinuous Galerkin methods and for this case there is a very large literature $[10,12,27,28]$, to name a few. In particular the mass matrix is block diagonal allowing for high order explicit time stepping, this however is no longer the case for IMEX methods where the system matrix associated to diffusion couples globally. Moreover if the target application is the incompressible Navier-Stokes' equations, the explicit methods are unsuitable and methods with many implicit stages may become costly. It is not clear that the analysis of [8] generalises to this case, since it is assumed there that the operator treated implicitly is symmetric and elliptic. For a second order scheme the RK IMEX method has three stages and is known to impose compatibility conditions on the exact solution for consistency to hold (see [8], Sect. 3, for a discussion in the case of convection-diffusion equations). Finite element-IMEX methods with extrapolation were considered as early as 1970 by Douglas and Dupont [15], however, they considered diffusion dominated problems. Implicit-explicit methods with extrapolation for the incompressible Navier-Stokes equations and backward differentiation used for time discretisation were analysed in [6], and some other multi-step IMEX methods together with the local Galerkin method was considered in [25], but, also here the mesh Peclet number was assumed to be small. In many applications, such as large eddy simulation or under resolved DNS, it may not be practical to make the Peclet number small, nevertheless for such problems, in which convective effects are strong, the use of IMEX schemes is very attractive, since the nonlinearity and stabilization terms are handled explicitly, whereas the velocity-pressure coupling, which is linear can be solved implicitly using optimized methods for saddle point problems, or pressure projection methods. We refer to [11] for work on fractional step methods in the high Peclet regime and to [21] for a computational study of underresolved turbulence using the type of methods that we analyse below.

As a first step towards IMEX schemes for the equations of incompressible flow we will in this paper consider the convection-diffusion equation and analyse some known IMEX schemes with respect to their stability properties for varying mesh Peclet number. For an IMEX scheme to be stable for high mesh Peclet number it has to degenerate to a stable explicit scheme in the limit of vanishing diffusion. Such time integrator are typically characterized by nontrivial imaginary stability boundary. Examples are given by Adams-Bashforth (AB) integrators of higher order such as AB3, AB4, AB7 and AB8 (see [16]). Unfortunately IMEX schemes, where the convection is treated explicitly using extrapolation in methods popular for the solution of incompressible flow problems such as the Crank-Nicolson scheme or the second order backward differentiation scheme, do not enjoy this property. Indeed in this case the limit schemes are the second order Adams-Bashforth scheme for pure transport and the extrapolated Gear scheme [24] (BDF2 with extrapolated convection). Both have trivial imaginary stability boundary and would therefore seem unsuitable candidates for Peclet robust IMEX methods. Nevertheless in this contribution we will consider these two schemes with a finite element space discretization stabilized using continuous interior penalty. Observe that the explicit treatment of the stabilization is appealing since it avoids having to handle the extended stencil on the level of the linear solver [9]. We use energy methods to prove that they are stable, irrespective of the Peclet number, under suitable CFL conditions. The stability however depends on the space discretization. Both the polynomial order of the approximation space and the stabilization of the convection operator come into play. Using the additional stability of the gradient penalty operator we show optimal error estimates for the material derivative and $L^{2}$-error error estimates with the classical order $O\left(h^{p+\frac{1}{2}}\right)$ (where $p$ is the polynomial degree), which is known to be the best that can be obtained for continuous FEM in the general case. Observe also that since our results are robust with respect to the Peclet number they remain valid for the case of vanishing diffusion, i.e. the pure transport equation. Therefore, the present work also gives the first analysis of these explicit methods together with stabilized FEM for the transport equation. 
We only consider the case of continuous approximation spaces herein, but the analysis carries over to the case of discontinuous Galerkin symmetric interior penalty methods, with upwind fluxes in a straightforward fashion (we refer to [10] for a uniform treatment of continuous and discontinuous Galerkin methods in the case of explicit Runge-Kutta methods).

The outline of the paper is as follows. In the next section we introduce our model problem, define the finite element spaces and prove some technical results. In Section 3.1 we introduce the BDF2-IMEX method and derive stability results in all regimes and for all polynomial orders. This allows us to prove a priori error estimates in Section 4. Here we consider the high Peclet regime only and derive error estimates for the $L^{2}$-error at final time and the error in the material derivative over the space-time domain. In Section 5 we introduce the Crank-Nicolson IMEX scheme and prove stability estimates in all regime and for all polynomial orders. Note that it is then straightforward to derive similar error estimates as for the BDF2-IMEX scheme in the high Peclet regime for the Crank-Nicolson scheme and also optimal estimates in $l^{2}\left(0, T ; H^{1}(\Omega)\right)$, or $l^{\infty}\left(0, T ; L^{2}(\Omega)\right)$ for both methods, but to keep the length of the paper reasonable these results have not been included here. The paper finishes with some numerical experiments (Sect. 6), validating the theory and showing the robustness of the methods in the presence of non-smooth data.

\section{Preliminaries}

\subsection{Convection-diffusion problem}

Let $\Omega \subset \mathbb{R}^{d}, d=1,2,3$ be an open polygonal domain with boundary $\partial \Omega$ and outward pointing normal $n$. Let $I:=(0, T)$ and denote the space time domain by $Q=\Omega \times I$. We consider the convection diffusion equation,

$$
\begin{aligned}
\partial_{t} u+\beta \cdot \nabla u-\mu \Delta u & =f & & \text { in } Q \\
u(\cdot, 0) & =u_{0} & & \text { in } \Omega \\
u & =0 & & \text { on } \partial \Omega .
\end{aligned}
$$

Here $f \in L^{2}(\Omega), u_{0} \in H_{0}^{1}(\Omega), \beta \in\left[H_{0}^{1}(\Omega) \cap W^{1, \infty}(\Omega)\right]^{d}$, with $\nabla \cdot \beta=0,\left.\beta \cdot n\right|_{\partial \Omega}=0$. This is a parabolic problem and it is known to admit a unique solution in $L^{2}\left(0, T ; H_{0}^{1}(\Omega)\right) \cap L^{\infty}\left(0, T ; L^{2}(\Omega)\right)$. We define the forms

$$
(u, v)_{\Omega}:=\int_{\Omega} u v \mathrm{~d} x, \quad c(w, v):=\int_{\Omega} \beta \cdot \nabla w v \mathrm{~d} x, \quad a(w, v):=\int_{\Omega} \mu \nabla w \cdot \nabla v \mathrm{~d} x .
$$

Assuming sufficient smoothness of the solution the equation may then be cast on the weak formulation,

$$
\begin{aligned}
\left(\partial_{t} u, v\right)_{\Omega}+c(u, v)+a(u, v) & =(f, v)_{\Omega}, \quad \forall v \in H_{0}^{1}(\Omega), t>0, \\
(u(\cdot, 0), v)_{\Omega} & =\left(u_{0}, v\right)_{\Omega}, \quad \forall v \in H_{0}^{1}(\Omega) .
\end{aligned}
$$

We will use the following two norms $\|\cdot\|^{2}=(\cdot, \cdot)_{\Omega}$ and $\|v\|_{\infty}=\sup _{x \in \bar{\Omega}}|v(x)|$.

\subsection{Finite element spaces and bilinear forms}

Let $\left\{\mathcal{T}_{h}\right\}_{h}$ denote a family of shape regular, quasi uniform, triangulation of $\Omega$ into simplices. The set of interior faces of $\mathcal{T}_{h}$ will be denoted $\mathcal{F}$. Let $V_{h}$ denote the space of continuous finite element functions of polynomial degree less than or equal to $p$ :

$$
V_{h}:=\left\{v \in H^{1}(\Omega): v \in \mathcal{P}_{p}(T), \forall T \in \mathcal{T}_{h}\right\} .
$$

We also consider the space with homogenuous boundary conditions $\stackrel{\circ}{V}_{h}=H_{0}^{1}(\Omega) \cap V_{h}$. We let $\pi_{h}$ be the $L^{2}$ projection onto $\stackrel{\circ}{V}_{h}$ given by

$$
\left(\pi_{h} w, v_{h}\right)_{\Omega}=\left(w, v_{h}\right)_{\Omega} \quad \forall v_{h} \in \stackrel{\circ}{V}_{h} .
$$


We recall the following approximation estimate that holds on quasi-uniform meshes

$$
\left\|u-\pi_{h} u\right\|+h\left\|\nabla\left(u-\pi_{h} u\right)\right\| \leq C h^{p+1}|u|_{H^{p+1}(\Omega)} .
$$

We will also make use of the piece-wise constant space

$$
W_{h}:=\left\{v \in L^{2}(\Omega): v \in \mathcal{P}_{0}(T), \forall T \in \mathcal{T}_{h}\right\} .
$$

We let $P_{0}: L^{2}(\Omega) \rightarrow W_{h}$ be the $L^{2}$ orthogonal projection:

$$
\left(P_{0} w, v_{h}\right)_{\Omega}=\left(w, v_{h}\right)_{\Omega} \quad \forall v_{h} \in W_{h} .
$$

In order to stabilize a FEM we need the following bilinear form (see e.g. [7])

$$
s\left(w_{h}, v_{h}\right):=\sum_{F \in \mathcal{F}} \int_{F} h_{F}^{2}\left(|\beta \cdot n|+\varepsilon_{\beta^{\perp}}\right) \llbracket \nabla w \rrbracket \cdot \llbracket \nabla v \rrbracket \mathrm{d} s,
$$

where we introduce the jump of the gradient

$$
\left.\llbracket \nabla w \rrbracket\right|_{F}:=\lim _{\epsilon \rightarrow 0^{+}} \nabla w\left(x-\epsilon n_{T}\right) \cdot n_{T}+\nabla w\left(x-\epsilon n_{T^{\prime}}\right) \cdot n_{T^{\prime}}, \text { with } x \in F \quad \text { and } F=\bar{T} \cap \bar{T}^{\prime}
$$

and $\varepsilon_{\beta^{\perp}} \geq 0$ is a coefficient that introduces some weakly consistent cross wind diffusion when non-zero. We will also apply the jump to scalar quantities below in which case it is defined by

$$
\left.\llbracket w \rrbracket\right|_{F}=\lim _{\epsilon \rightarrow 0^{+}} w\left(x-\epsilon n_{F}\right)-w\left(x+\epsilon n_{F}\right) \text { with } x \in F
$$

where $n_{F}$ is a fixed but arbitrary normal to the face $F$. We may then define the semi-norm

$$
|v|_{s}:=s(v, v)^{\frac{1}{2}}
$$

We let $\beta_{0}$ be the Raviart-Thomas projection of $\beta$ to the lowest order Raviart-Thomas space. Since $\nabla \cdot \beta=0$ we have that $\beta_{0}$ is piecewise constant. Thus, we have

$$
\left\|\beta-\beta_{0}\right\|_{\infty} \leq C h\|\nabla \beta\|_{\infty} .
$$

Then recall a critical approximation result that exhibits the importance of the stabilization term, this follows from the local estimate of Lemma 5.3 from [7]:

$$
\inf _{v_{h} \in V_{h}}\left\|\beta_{0} \cdot \nabla w_{h}-v_{h}\right\|^{2} \leq C \sum_{F \in \mathcal{F}} h_{F}\left\|\llbracket \beta_{0} \cdot \nabla w_{h} \rrbracket\right\|_{F}^{2}, \quad \forall w_{h} \in V_{h} .
$$

Note that since $\left.\beta_{0} \cdot n\right|_{\partial \Omega}=\left.\beta \cdot n\right|_{\partial \Omega}=0$ we have $\left.\beta_{0} \cdot \nabla w_{h}\right|_{\partial \Omega}=0$ when $w_{h} \in \stackrel{\circ}{V}_{h}$ and therefore (2.7) holds taking the infimum over the space $\stackrel{\circ}{h}_{h}$, i.e.

$$
\inf _{v_{h} \in \grave{V}_{h}}\left\|\beta_{0} \cdot \nabla w_{h}-v_{h}\right\|^{2} \leq C \sum_{F \in \mathcal{F}} h_{F}\left\|\llbracket \beta_{0} \cdot \nabla w_{h} \rrbracket\right\|_{F}^{2}, \quad \forall w_{h} \in \stackrel{\circ}{V}_{h} .
$$

Using (2.7) together with (2.6), we have the following lemma:

Lemma 2.1. It holds,

$$
\inf _{v_{h} \in \dot{V}_{h}}\left\|\beta \cdot \nabla w_{h}-v_{h}\right\| \leq C\left(\|\nabla \beta\|_{\infty}\left\|w_{h}\right\|+\left(\frac{\|\beta\|_{\infty}}{h}\right)^{\frac{1}{2}}\left|w_{h}\right|_{s}\right), \quad \forall w_{h} \in \stackrel{\circ}{V}_{h} .
$$


Proof. We first add and subtract $\beta_{0}$ and apply the triangle inequality

$$
\left\|\beta \cdot \nabla w_{h}-v_{h}\right\| \leq\left\|\left(\beta-\beta_{0}\right) \cdot \nabla w_{h}\right\|+\left\|\beta_{0} \cdot \nabla w_{h}-v_{h}\right\| .
$$

Then using (2.6), an inverse inequality and (2.7)

$$
\inf _{v_{h} \in \cup_{h}}\left\|\beta \cdot \nabla w_{h}-v_{h}\right\| \leq C\left(\|\nabla \beta\|_{\infty}\left\|w_{h}\right\|+\left(\sum_{F \in \mathcal{F}} h_{F}\left\|\llbracket \beta_{0} \cdot \nabla w_{h} \rrbracket\right\|_{F}^{2}\right)^{\frac{1}{2}}\right) .
$$

Adding and subtracting $\beta$ in the second term and using a trace inequality followed by (2.6),

$$
\begin{aligned}
\sum_{F \in \mathcal{F}} h_{F}\left\|\llbracket \beta_{0} \cdot \nabla w_{h} \rrbracket\right\|_{F}^{2} & \leq C\left(\left\|\left(\beta-\beta_{0}\right) \cdot \nabla w_{h}\right\|^{2}+\|\nabla \beta\|_{\infty}^{2}\left\|w_{h}\right\|^{2}+\sum_{F \in \mathcal{F}} h_{F}\left\|\llbracket \beta \cdot \nabla w_{h} \rrbracket\right\|_{F}^{2}\right) \\
& \leq C\left(\|\nabla \beta\|_{\infty}^{2}\left\|w_{h}\right\|^{2}+\sum_{F \in \mathcal{F}} h_{F}\left\|\llbracket \beta \cdot \nabla w_{h} \rrbracket\right\|_{F}^{2}\right) .
\end{aligned}
$$

Using the continuity of $w_{h}$ in the last term of the right hand side we see that

$$
\left\|\llbracket \beta \cdot \nabla w_{h} \rrbracket\right\|_{F}=\left\|\beta \cdot n \llbracket \nabla w_{h} \rrbracket\right\|_{F} \leq\|\beta\|_{\infty}^{\frac{1}{2}}\left\||\beta \cdot n|^{\frac{1}{2}} \llbracket \nabla w_{h} \rrbracket\right\|_{F} .
$$

Hence, we have shown (2.9).

We can then define the stabilised convection form

$$
c_{h}\left(w_{h}, v_{h}\right):=c\left(w_{h}, v_{h}\right)+\gamma s\left(w_{h}, v_{h}\right) .
$$

Introducing $\tau$ as the time step size, we also define Courant number Co that will either be the standard hyperbolic CFL, Co $:=\left(\|\beta\|_{\infty}+1\right) \frac{\tau}{h}$, or a slightly stronger 4/3-Courant number (see $[10,27]$ where it was used in the context of second order Runge-Kutta methods), $\mathrm{Co}_{4 / 3}:=\tau\left(\|\beta\|_{\infty} / h\right)^{\frac{4}{3}}$, that will apply for finite element spaces or polynomial degrees higher than 1 . Observe that Co is a free parameter that can be made as small as we like by making $\tau$ small relative to $h$ and $\beta$. The crucial point is that certain time residual terms from the convection term can be made as small as necessary by fixing Co to be small enough. This is expressed in boundedness properties of the convection and the associated stabilization that we now summarize. First note that by the skew symmetry of the convection we have the positivity

$$
\gamma|v|_{s}^{2}=c_{h}(v, v), \quad \forall v \in H_{0}^{1}(\Omega) \cap H^{\frac{3}{2}+\epsilon}(\Omega)+\stackrel{\circ}{V}_{h},
$$

and by skew-symmetry followed by the Cauchy-Schwarz inequality, an inverse inequality and the definition of Co we have the positivity

$$
\tau c\left(v, w_{h}\right) \leq C_{i} \mathrm{Co}\|v\|\left\|w_{h}\right\| \quad \forall v \in H_{0}^{1}(\Omega), w_{h} \in V_{h},
$$

where $C_{i}$ the constant of an inverse inequality. Similarly for the stabilisation norm we have the bound

$$
\tau^{\frac{1}{2}}\left|w_{h}\right|_{s} \leq C_{i} \mathrm{Co}^{\frac{1}{2}}\left\|w_{h}\right\| \quad \forall w_{h} \in V_{h}
$$

For the analysis we introduce a projection operator $C_{h}: H^{1}(\Omega) \mapsto \stackrel{\circ}{V_{h}}$ defined by

$$
\left(C_{h} v, v_{h}\right)_{\Omega}=c\left(v, v_{h}\right), \quad \forall v_{h} \in \stackrel{\circ}{V}_{h} .
$$

Note that by the definition of $c,\left(C_{h} w_{h}, v_{h}\right)_{\Omega}=-\left(w, C_{h} v_{h}\right)_{\Omega}$ for $w_{h}, v_{h} \in \stackrel{\circ}{V}_{h}$. Using (2.11) it is straightforward to show the following bound for the operator $C_{h}$. 
Lemma 2.2. It holds,

$$
\tau\left\|C_{h} v\right\| \leq C_{i} \tau^{\frac{1}{4}} \mathrm{Co}_{4 / 3}^{\frac{3}{4}}\|v\|, \quad \forall v \in H^{1}(\Omega)
$$

Proof.

$$
\tau^{2}\left(C_{h} v, C_{h} v\right)=\tau^{2} c\left(v, C_{h} v\right)=-\tau^{2} c\left(C_{h} v, v\right) \leq \tau^{2} h^{-1}\|\beta\|_{\infty} C_{i}\left\|C_{h} v\right\|\|v\| .
$$

Therefore,

$$
\tau\left\|C_{h} v\right\| \leq \tau h^{-1}\|\beta\|_{\infty} C_{i}\|v\|,
$$

but $\tau=\tau^{\frac{1}{4}} \mathrm{Co}_{4 / 3}^{\frac{3}{4}} h /\|\beta\|_{\infty}$ by which the claim follows.

We also notice that

$$
\tau\left\|C_{h} v\right\| \leq C_{i} \mathrm{Co}\|v\| .
$$

\subsection{Operators for time discretization}

We define the second order backward differentiation operator

$$
D_{\tau} v^{n+1}:=\frac{3 v^{n+1}-4 v^{n}+v^{n-1}}{2 \tau} .
$$

We recall the second order extrapolation $\tilde{v}^{n+1}=2 v^{n}-v^{n-1}$, and the increment operator $\delta$ such that $\delta v^{n+1}:=$ $v^{n+1}-v^{n}$. Observe that there holds

$$
\tilde{v}^{n+1}-v^{n+1}=\delta v^{n}-\delta v^{n+1}=-\delta \delta v^{n+1} .
$$

We also recall that

$$
\tau D_{\tau} v^{n+1}=\delta v^{n+1}+\frac{1}{2} \delta \delta v^{n+1}
$$

Finally, we also observe that

$$
\left\|\delta^{m} v^{n}\right\| \leq 2 \sum_{i=0}^{m}\left\|v^{n-i}\right\|, \quad m=1,2, \quad \text { and } 2 \leq n \leq N .
$$

As we will describe in a later section, for the Crank-Nicolson method the approximation of the time derivative is given by the scaled increment operator $\tau^{-1} \delta v^{n+1}$. The extrapolation is taken to the time level $t^{n+1 / 2}$, in order to approximate the central difference in time that is the key feature of the Crank-Nicolson scheme, $\hat{v}^{n+1}=\frac{3}{2} v^{n}-\frac{1}{2} v^{n-1}$.

For the time discretization part of the error analysis we need some well known results on truncation error analysis of finite difference operators that we collect in the following proposition for future reference. These results are standard and can be found for instance in the monograph [23], but for completeness we sketch the proofs.

Proposition 2.3. Let $u^{n}:=u\left(t^{n}\right)$ and $y:=\beta \cdot \nabla u$ then there holds

$$
\begin{gathered}
\left\|D_{\tau} u^{n+1}-\partial_{t} u^{n+1}\right\|^{2} \leq C \tau^{3}\left\|u_{t t t}\right\|_{L^{2}\left(t^{n-1}, t^{n+1} ; L^{2}(\Omega)\right)}^{2} \\
\left\|\beta\left(t^{n+1}\right) \cdot \nabla u^{n+1}-\tilde{y}^{n+1}\right\|^{2} \leq C \tau^{3}\left\|(\beta \cdot \nabla u)_{t t}\right\|_{L^{2}\left(t^{n-1}, t^{n+1} ; L^{2}(\Omega)\right)}^{2} .
\end{gathered}
$$

Proof. We first consider the bound (2.19). We wish to bound

$$
\left\|D_{\tau} u^{n+1}-\partial_{t} u^{n+1}\right\|
$$


Using Taylor development we may write

$$
u(t)=u^{n-1}+\left(t-t^{n-1}\right) \partial_{t} u^{n-1}+\frac{1}{2}\left(t-t^{n-1}\right)^{2} \partial_{t}^{2} u^{n-1}+\underbrace{\frac{1}{2} \int_{t_{n-1}}^{t}(t-s)^{2} u^{(3)}(s) \mathrm{d} s}_{R(t)}=Q(t)+R(t) .
$$

Deriving and evaluating at $t^{n+1}$ we see that

$$
Q^{\prime}(t)=\partial_{t} u^{n-1}+\left(t-t^{n-1}\right) \partial_{t}^{2} u^{n-1}, \quad Q^{\prime}\left(t^{n+1}\right)=\partial_{t} u^{n-1}+2 \tau \partial_{t}^{2} u^{n-1}
$$

We also have

$$
\tau^{-1} \delta Q^{n+1}=\partial_{t} u^{n-1}+\frac{1}{2 \tau}\left(\left(t^{n+1}-t^{n-1}\right)^{2}-\left(t^{n}-t^{n-1}\right)^{2}\right) \partial_{t}^{2} u^{n-1}=\partial_{t} u^{n-1}+\frac{3 \tau}{2} \partial_{t}^{2} u^{n-1}
$$

and

$$
\begin{aligned}
\tau^{-1} \delta^{2} Q^{n+1}= & \underbrace{\left(\left(t^{n+1}-t^{n-1}\right)-2\left(t^{n}-t^{n-1}\right)\right) \partial_{t} u^{n-1}}_{=0} \\
& +\frac{1}{2 \tau}\left(\left(t^{n+1}-t^{n-1}\right)^{2}-2\left(t^{n}-t^{n-1}\right)^{2}\right) \partial_{t}^{2} u^{n-1}=\tau^{2} \partial_{t}^{2} u^{n-1} .
\end{aligned}
$$

Using (2.17) we see that

$$
D_{\tau} Q^{n+1}=\partial_{t} u^{n-1}+\frac{3 \tau}{2} \partial_{t}^{2} u^{n-1}+\frac{\tau}{2} \partial_{t}^{2} u^{n-1}=\partial_{t} u^{n-1}+2 \tau \partial_{t}^{2} u^{n-1}=Q^{\prime}\left(t^{n+1}\right) .
$$

Therefore

$$
\left\|D_{\tau} u^{n+1}-\partial_{t} u^{n+1}\right\|^{2}=\|\underbrace{D_{\tau} Q-Q^{\prime}}_{=0}+D_{\tau} R^{n+1}-\partial_{t} R^{n+1}\|^{2} \leq \frac{C}{\tau^{2}} \sum_{k=n-1}^{n+1}\left\|R^{k}\right\|^{2}+2\left\|\partial_{t} R^{n+1}\right\|^{2} .
$$

By the definition of $R$ and the Cauchy-Schwarz inequality:

$$
\frac{1}{\tau^{2}} \sum_{k=n-1}^{n+1}\left\|R^{k}\right\|^{2} \leq C \tau^{3} \int_{t^{n-1}}^{t^{n+1}}\left\|u^{(3)}\right\|^{2} \mathrm{~d} s
$$

Finally

$$
\partial_{t} R^{n+1}=\int_{t^{n-1}}^{t^{n+1}}\left(t^{n}-s\right) u^{(3)}(s) \mathrm{d} s
$$

and therefore in a similar fashion

$$
\left\|\partial_{t} R^{n+1}\right\|^{2} \leq \tau^{3} \int_{t^{n-1}}^{t^{n+1}}\left\|u^{(3)}\right\|^{2} \mathrm{~d} s
$$

which gives (2.19). The result (2.20) easily follows after we apply the Cauchy-Shwarz inequality

$$
\left\|y^{n+1}-\tilde{y}^{n+1}\right\|^{2}=\left\|\int_{t^{n-1}}^{t^{n}} \int_{t}^{t+\tau} y_{t t}(s) \mathrm{d} s \mathrm{~d} t\right\|^{2} \leq \tau^{3} \int_{t_{n-1}}^{t^{n+1}}\left\|y_{t t}(s)\right\|^{2} \mathrm{~d} s .
$$

We will also make use of the following summation by parts formulas. 
Lemma 2.4. Let $r(\cdot, \cdot)$ denote a bilinear form on $V_{h} \times V_{h}$. Then the following summation by parts formulas holds

$$
\sum_{n=1}^{N-1} r\left(\delta v^{n+1}, w^{n+1}\right)=r\left(v^{N}, w^{N}\right)-r\left(v^{1}, w^{2}\right)-\sum_{n=2}^{N-1} r\left(v^{n}, \delta w^{n+1}\right) .
$$

Proof. We write

$$
\begin{aligned}
\sum_{n=1}^{N-1} r\left(\delta v^{n+1}, w^{n+1}\right) & =\sum_{n=1}^{N-1} r\left(v^{n+1}, w^{n+1}\right)-\sum_{n=1}^{N-1} r\left(v^{n}, w^{n+1}\right) \\
& =r\left(v^{N}, w^{N}\right)+\sum_{n=2}^{N-1} r\left(v^{n}, w^{n}\right)-\sum_{n=1}^{N-1} r\left(v^{n}, w^{n+1}\right) \\
& =r\left(v^{N}, w^{N}\right)-r\left(v^{1}, w^{2}\right)-\sum_{n=2}^{N-1} r\left(v^{n}, \delta w^{n+1}\right) .
\end{aligned}
$$

Finally, we will need the following discrete simple form of the discrete Gronwall's inequality.

Proposition 2.5. Let $\left\{\phi_{n}\right\}$ be a sequence of non-negative numbers and let $\psi$ and $\eta$ be non-negative numbers such that

$$
\phi_{n} \leq \psi+\eta \sum_{i=1}^{n} \phi_{i}
$$

Then, the following estimate holds

$$
\phi_{N} \leq\left(1+N \eta e^{\eta N}\right) \psi
$$

\section{The BDF2-IMEX Method}

We may write the BDF2-IMEX finite element method as follows.

Find $u_{h}^{n+1} \in \stackrel{\circ}{V}_{h}$ such that for $n \geq 1$,

$$
\left(D_{\tau} u_{h}^{n+1}, v_{h}\right)_{\Omega}+c_{h}\left(\tilde{u}_{h}^{n+1}, v_{h}\right)+a\left(u_{h}^{n+1}, v_{h}\right)=L^{n+1}\left(v_{h}\right), \quad \forall v_{h} \in \stackrel{\circ}{V}_{h},
$$

where $u_{h}^{0}, u_{h}^{1}$ are given. Here $\left\{L^{n}\right\}$ are a bounded linear operator on $\stackrel{\circ}{V}_{h}$.

\subsection{Stability of BDF2-IMEX}

In the diffusion dominated $(\mathrm{Pe}<1)$ regime the BDF2-IMEX method is stable under the standard hyperbolic CFL condition. In this section we prove in addition to this, that BDF2-IMEX the method is stable indepdendent of the Peclet number with a standard hyperbolic CFL condition when $p=1$ and under the $4 / 3$-CFL when $p>1$.

Let us define some norms. We start by defining the natural dissipation of the spatial variables.

$$
E(v)^{2}:=\gamma|v|_{s}^{2}+\left\|\mu^{\frac{1}{2}} \nabla v\right\|^{2}
$$

We see that $E(v)^{2}=c_{h}(v, v)+a(v, v)$ when $v \in \stackrel{\circ}{V}_{h}$. An immediate consequence of (2.12) and an inverse inequality is that for all $v \in V_{h}$,

$$
\gamma|v|_{s}^{2} \leq \frac{C \gamma\left(\|\beta\|_{\infty}+1\right)}{h}\|v\|^{2},
$$




$$
\left\|\mu^{\frac{1}{2}} \nabla v\right\|^{2} \leq \frac{C \mu}{h^{2}}\|v\|^{2}
$$

Hence,

$$
\tau E(v)^{2} \leq C\left(\gamma \mathrm{Co}+\frac{\mathrm{Co}}{\mathrm{Pe}}\right)\|v\|^{2},
$$

where we recall that the definition of the Peclet number Pe:

$$
\mathrm{Pe}:=\frac{\|\beta\|_{\infty} h}{\mu} .
$$

For a linear operator $L$ defined for $\stackrel{\circ}{V}_{h}$ we define

$$
\|L\|_{h}=\sup _{v \in \grave{V}_{h}} \frac{L(v)}{\sqrt{E(v)^{2}+\|v\|^{2}}} .
$$

We introduce the triple norm, measuring the dissipation in the system,

$$
\|v\| \|^{2}:=\sum_{n=1}^{N-1}\left(\tau E\left(v^{n+1}\right)^{2}+\frac{1}{4}\left\|v^{n+1}-\tilde{v}^{n+1}\right\|^{2}\right) .
$$

The following elementary relationship will be useful.

$$
\tau\left(D_{\tau} v^{n+1}, v^{n+1}\right)_{\Omega}=\frac{1}{4}\left(\left\|v^{n+1}\right\|^{2}+\left\|\tilde{v}^{n+2}\right\|^{2}-\left(\left\|v^{n}\right\|^{2}+\left\|\tilde{v}^{n+1}\right\|^{2}\right)+\left\|v^{n+1}-\tilde{v}^{n+1}\right\|^{2}\right) .
$$

\subsection{The case $\mathrm{Pe} \leq 1$ for $p \geq 1$ and the case $\mathrm{Pe}>1$ for $p=1$}

Before proving stability we prove an auxiliary result which will be helpful for the case $p=1$ and $\mathrm{Pe}>1$. This result says that the discrete time derivative minus its projection on the element wise constants can be controlled using the dissipative terms in space, primarily the stabilization. This can then be applied in the stability analysis, reducing the need to apply inverse inequalities to control the convective term, by using the control of the stabilizing term and hence achieving stability under the standard hyperbolic CFL condition.

Lemma 3.1. Let $p=1$ and let $u_{h}$ solve (3.1) then the following estimate holds

$$
\begin{aligned}
\left\|\tau D_{\tau} u_{h}^{n+1}-P_{0}\left(\tau D_{\tau} u_{h}^{n+1}\right)\right\| \leq & C \sqrt{\tau} \sqrt{\operatorname{Co}} K E\left(u_{h}^{n+1}\right)+C \tau\|\nabla \beta\|_{\infty}\left\|\tilde{u}_{h}^{n+1}\right\|+C \operatorname{Co}\left\|\tilde{u}_{h}^{n+1}-u_{h}^{n+1}\right\| \\
& +C \sqrt{\tau}\left(\frac{\sqrt{\mathrm{Co}}}{\sqrt{\mathrm{Pe}}}+\sqrt{\operatorname{Co}} \sqrt{\gamma}+1\right)\left\|L^{n+1}\right\|_{h},
\end{aligned}
$$

where

$$
K(\gamma, \mathrm{Pe}):=\left(\frac{1}{\mathrm{Pe}}+\sqrt{\gamma}+\frac{1}{\sqrt{\gamma}}\right)
$$

Proof. Let $y_{h}=\tau D_{\tau} u_{h}^{n+1}$ and then we have by (3.1)

$$
\begin{aligned}
\left\|y_{h}-P_{0} y_{h}\right\|^{2}= & \left(y_{h}, y_{h}-P_{0}\left(y_{h}\right)\right)_{\Omega} \\
= & \left(y_{h}, \pi_{h}\left(y_{h}-P_{0}\left(y_{h}\right)\right)\right)_{\Omega} \\
= & -\tau c_{h}\left(\tilde{u}_{h}^{n+1}, \pi_{h}\left(y_{h}-P_{0}\left(y_{h}\right)\right)\right)-\tau a\left(u_{h}^{n+1}, \pi_{h}\left(y_{h}-P_{0}\left(y_{h}\right)\right)\right) \\
& +\tau L^{n+1}\left(\pi_{h}\left(y_{h}-P_{0}\left(y_{h}\right)\right)\right) .
\end{aligned}
$$


We use the Cauchy-Schwarz inequality followed inverse estimates to bound the symmetric terms

$$
\begin{aligned}
-\tau a\left(u_{h}^{n+1}, \pi_{h}\left(y_{h}-P_{0}\left(y_{h}\right)\right)\right) & \leq C \frac{\sqrt{\mu} \tau}{h}\left\|\sqrt{\mu} \nabla u_{h}^{n+1}\right\|\left\|y_{h}-P_{0}\left(y_{h}\right)\right\| \\
& \leq C \frac{\sqrt{\tau \mathrm{Co}}}{\sqrt{\mathrm{Pe}}}\left\|\sqrt{\mu} \nabla u_{h}^{n+1}\right\|\left\|y_{h}-P_{0}\left(y_{h}\right)\right\| .
\end{aligned}
$$

For the stabilization we apply $(2.12)$

$$
-\tau \gamma s\left(\tilde{u}_{h}^{n+1}, \pi_{h}\left(y_{h}-P_{0}\left(y_{h}\right)\right)\right) \leq C \sqrt{\tau} \sqrt{\gamma \operatorname{Co}} \sqrt{\gamma}\left|\tilde{u}_{h}^{n+1}\right|_{s}\left\|y_{h}-P_{0}\left(y_{h}\right)\right\| .
$$

Next we bound $\tau L^{n+1}\left(\pi_{h}\left(y_{h}-P_{0}\left(y_{h}\right)\right)\right)$ using (3.4) and (3.3).

$$
\begin{aligned}
\tau L^{n+1}\left(\pi_{h}\left(y_{h}-P_{0}\left(y_{h}\right)\right)\right) & \leq \tau\left\|L^{n+1}\right\|_{h} \sqrt{E\left(\pi_{h}\left(y_{h}-P_{0}\left(y_{h}\right)\right)\right)^{2}+\left\|\pi_{h}\left(y_{h}-P_{0}\left(y_{h}\right)\right)\right\|^{2}} \\
& \leq C \sqrt{\tau}\left(\frac{\sqrt{\mathrm{Co}}}{\sqrt{\mathrm{Pe}}}+\sqrt{\gamma \mathrm{Co}}+\sqrt{\tau}\right)\left\|L^{n+1}\right\|_{h}\left\|y_{h}-P_{0}\left(y_{h}\right)\right\| .
\end{aligned}
$$

To bound the first term we observe that by (3.7) it only remains to bound the contribution from the form $c$.

$$
\begin{aligned}
-\tau c\left(\tilde{u}_{h}^{n+1}, \pi_{h}\left(y_{h}-P_{0}\left(y_{h}\right)\right)\right)= & -\tau\left(\left(\beta-\beta_{0}\right) \cdot \nabla \tilde{u}_{h}^{n+1}, \pi_{h}\left(y_{h}-P_{0}\left(y_{h}\right)\right)\right)_{\Omega} \\
& -\tau\left(\beta_{0} \cdot \nabla \tilde{u}_{h}^{n+1}-w_{h}^{n+1},\left(I-\pi_{h}\right)\left(y_{h}-P_{0}\left(y_{h}\right)\right)\right)_{\Omega} .
\end{aligned}
$$

Here $w_{h} \in \stackrel{\circ}{V}_{h}$ is arbitrary. Note that we crucially used that $p=1$ which implies that $\beta_{0} \cdot \nabla \tilde{u}_{h}^{n+1} \in W_{h}$.

Hence, using (2.6) and (2.7) we obtain

$$
\begin{aligned}
-\tau c\left(\tilde{u}_{h}^{n+1}, \pi_{h}\left(y_{h}-P_{0}\left(y_{h}\right)\right)\right) & \leq C \tau\left(\|\nabla \beta\|_{\infty}\left\|\tilde{u}_{h}^{n+1}\right\|+\|\beta\|_{\infty}^{1 / 2} h^{-1 / 2}\left|\tilde{u}_{h}^{n+1}\right|_{s}\right)\left\|y_{h}-P_{0}\left(y_{h}\right)\right\| \\
& \leq C \sqrt{\tau}\left(\sqrt{\tau}\|\nabla \beta\|_{\infty}\left\|\tilde{u}_{h}^{n+1}\right\|+\sqrt{\operatorname{Co}} \frac{1}{\sqrt{\gamma}} \sqrt{\gamma}\left|\tilde{u}_{h}^{n+1}\right|_{s}\right)\left\|y_{h}-P_{0}\left(y_{h}\right)\right\| .
\end{aligned}
$$

Finally, by the triangle inequality and (2.12) we have the bound $\left|\tilde{u}_{h}^{n+1}\right|_{s} \leq\left|u_{h}^{n+1}\right|_{s}+\frac{C \sqrt{\mathrm{Co}_{0}}}{\sqrt{\tau}}\left\|u_{h}^{n+1}-\tilde{u}_{h}^{n+1}\right\|$. Combining the above inequalities gives the result.

We will use the following notation: We let $c_{L}=0$ if $L^{n} \equiv 0$ for every $n$ and $c_{L}=1$ otherwise. The following results prove stability under the standard hyperbolic CFL, in the low Peclet regime or, for piecewise affine approximation, in the high Peclet regime.

Theorem 3.2. Suppose that $T=N \tau$. Suppose that Co is chosen sufficiently small only depending on geometric constants of the mesh and $\gamma$. For $\left\{u_{h}^{n}\right\}$ solving (3.1) we have the following bounds:

If $\mathrm{Pe} \leq 1$ then for all $p \geq 1$,

$$
\left\|u_{h}^{N}\right\|^{2} \leq\left(1+\frac{T c_{L}}{8} e^{\frac{T c_{L}}{8}}\right)\left(\left\|u_{h}^{0}\right\|^{2}+\left\|u_{h}^{1}\right\|^{2}+32 \tau \sum_{n=1}^{N-1}\left\|L^{n+1}\right\|_{h}^{2}\right) .
$$

If $\mathrm{Pe}>1$ and $p=1, \gamma>0$ then

$$
\left\|u_{h}^{N}\right\|^{2} \leq\left(\left\|u_{h}^{0}\right\|^{2}+\left\|u_{h}^{1}\right\|^{2}+\left\|u_{h}^{2}\right\|^{2}+9 \tau \sum_{n=1}^{N-1}\left\|L^{n+1}\right\|_{h}^{2}\right) M,
$$

where

$$
M=8\left(1+c T\left(\tau\|\nabla \beta\|_{\infty}^{2}+c_{L}\right) e^{c T\left(\tau\|\nabla \beta\|_{\infty}^{2}+c_{L}\right)}\right)
$$


Proof. We test equation (3.1) with $u_{h}^{n+1}$ and get

$$
\left(D_{\tau} u_{h}^{n+1}, u_{h}^{n+1}\right)_{\Omega}+c_{h}\left(\tilde{u}_{h}^{n+1}, u_{h}^{n+1}\right)+a\left(u_{h}^{n+1}, u_{h}^{n+1}\right)=L^{n+1}\left(u_{h}^{n+1}\right) .
$$

Thus, we see that

$$
\left(D_{\tau} u_{h}^{n+1}, u_{h}^{n+1}\right)_{\Omega}+c_{h}\left(u_{h}^{n+1}, u_{h}^{n+1}\right)+a\left(u_{h}^{n+1}, u_{h}^{n+1}\right)=c_{h}\left(u_{h}^{n+1}-\tilde{u}_{h}^{n+1}, u_{h}^{n+1}\right)+L^{n+1}\left(u_{h}^{n+1}\right) .
$$

Then by summing over $n=1, \ldots, N-1$, multiplying with $\tau$, and using (3.5), (2.10)

$$
\frac{1}{4}\left(\left\|u_{h}^{N}\right\|^{2}+\left\|\tilde{u}_{h}^{N+1}\right\|^{2}\right)-\frac{1}{4}\left(\left\|u_{h}^{1}\right\|^{2}+\left\|\tilde{u}_{h}^{2}\right\|^{2}\right)+\left\|\mid u_{h}\right\| \|^{2}=S_{1}+S_{2}+S_{3}
$$

where

$$
\begin{aligned}
S_{1} & :=\tau \sum_{n=1}^{N-1} c\left(u_{h}^{n+1}-\tilde{u}_{h}^{n+1}, u_{h}^{n+1}\right), \\
S_{2} & :=\tau \sum_{n=1}^{N-1} \gamma s\left(u_{h}^{n+1}-\tilde{u}_{h}^{n+1}, u_{h}^{n+1}\right), \\
S_{3} & :=\tau \sum_{n=1}^{N-1} L^{n+1}\left(u_{h}^{n+1}\right) .
\end{aligned}
$$

Let us estimate $S_{3}$. We have

$$
\begin{aligned}
S_{3} & \leq \tau \sum_{n=1}^{N-1}\left\|L^{n+1}\right\|_{h} \sqrt{E\left(u_{h}^{n+1}\right)^{2}+\left\|u_{h}^{n+1}\right\|^{2}} \\
& \leq 8 \tau \sum_{n=1}^{N-1}\left\|L^{n+1}\right\|_{h}^{2}+\frac{1}{32} \tau \sum_{n=1}^{N-1} E\left(u_{h}^{n+1}\right)^{2}+\frac{c_{L}}{32} \tau \sum_{n=1}^{N-1}\left\|u_{h}^{n+1}\right\|^{2} .
\end{aligned}
$$

Now we estimate $S_{2}$. Using the arithmetic-geometric mean inequality and inverse estimates we obtain

$$
S_{2} \leq \sqrt{\operatorname{Co}} \gamma \sum_{n=1}^{N-1}\left\|u_{h}^{n+1}-\tilde{u}_{h}^{n+1}\right\|^{2}+C \sqrt{\operatorname{Co}} \tau \sum_{n=1}^{N-1} \gamma\left|u_{h}^{n+1}\right|_{s}^{2} .
$$

Next we bound $S_{1}$. We consider two cases: $\mathrm{Pe}>1$ and $\mathrm{Pe} \leq 1$.

Case 1. Pe $\leq 1, p \geq 1$ : Using that $c\left(u_{h}^{n+1}-\tilde{u}_{h}^{n+1}, u_{h}^{n+1}\right)=-c\left(u_{h}^{n+1}, u_{h}^{n+1}-\tilde{u}_{h}^{n+1}\right)$ we obtain

$$
\begin{aligned}
S_{1} & \leq C \sqrt{\operatorname{Co}} \sqrt{\operatorname{Pe}} \sqrt{\tau} \sum_{n=1}^{N-1}\left\|u_{h}^{n+1}-\tilde{u}_{h}^{n+1}\right\|\left\|\sqrt{\mu} \nabla u_{h}^{n+1}\right\| \\
& \leq \sqrt{\operatorname{Co}} \sum_{n=1}^{N-1}\left\|u_{h}^{n+1}-\tilde{u}_{h}^{n+1}\right\|^{2}+C \sqrt{\operatorname{Co}} \tau \sum_{n=1}^{N-1}\left\|\sqrt{\mu} \nabla u_{h}^{n+1}\right\|^{2} .
\end{aligned}
$$

Thus, using (3.10) and the fact that Co is sufficiently small we obtain

$$
\frac{1}{4}\left(\left\|u_{h}^{N}\right\|^{2}+\left\|\tilde{u}_{h}^{N+1}\right\|^{2}\right)+\left.\frac{1}{2}\left\|u_{h}\right\|\right|^{2} \leq \frac{1}{4}\left(\left\|u_{h}^{1}\right\|^{2}+\left\|\tilde{u}_{h}^{2}\right\|^{2}\right)+8 \tau \sum_{n=1}^{N-1}\left\|L^{n+1}\right\|_{h}^{2}+\frac{c_{L}}{32} \tau \sum_{n=1}^{N-1}\left\|u_{h}^{n+1}\right\|^{2} .
$$

Using Gronwall's inequality (2.23) we have (3.8). 
Case 2. Pe $>1$ and $p=1$ : We use (2.16) and (2.22) to obtain

$$
S_{1}=\tau \sum_{n=1}^{N-1} c\left(\delta \delta u_{h}^{n+1}, u_{h}^{n+1}\right)=-\tau \sum_{n=2}^{N-1} c\left(\delta u_{h}^{n}, \delta u_{h}^{n+1}\right)+\tau\left(c\left(\delta u_{h}^{N}, u_{h}^{N}\right)-c\left(\delta u_{h}^{1}, u_{h}^{2}\right)\right) .
$$

Using that $c\left(\delta \delta u_{h}^{n+1}, \delta \delta u_{h}^{n+1}\right)=0=c\left(\delta u_{h}^{n+1}, \delta u_{h}^{n+1}\right)$ and (2.17) we have

$$
-c\left(\delta u_{h}^{n}, \delta u_{h}^{n+1}\right)=c\left(\delta \delta u_{h}^{n+1}, \delta u_{h}^{n+1}\right)=c\left(\delta \delta u_{h}^{n+1}, \tau D_{\tau} u_{h}^{n+1}\right) .
$$

Thus,

$$
S_{1}=\tau \sum_{n=2}^{N-1} c\left(\delta \delta u_{h}^{n+1}, \tau D_{\tau} u_{h}^{n+1}\right)+\tau\left(c\left(\delta u_{h}^{N}, u_{h}^{N}\right)-c\left(\delta u_{h}^{1}, u_{h}^{2}\right)\right) .
$$

We let $y_{h}^{n+1}=\tau D_{\tau} u_{h}^{n+1}$ and use the fact that $P_{0}\left(y_{h}^{n+1}\right)$ is in the kernel of the gradient operator followed by an inequality similar to (2.11), but applied elementwise, to see that

$$
\tau c\left(\delta \delta u_{h}^{n+1}, y_{h}^{n+1}\right)=-\tau c\left(y_{h}^{n+1}, \delta \delta u_{h}^{n+1}\right) \leq C \operatorname{Co}\left\|\delta \delta u_{h}^{n+1}\right\|\left\|y_{h}^{n+1}-P_{0}\left(y_{h}^{n+1}\right)\right\| .
$$

Thus, applying (3.6) we obtain

$$
\begin{aligned}
& \tau \sum_{n=2}^{N-1} c\left(\delta \delta u_{h}^{n+1}, \tau D_{\tau} u_{h}^{n+1}\right) \\
& \leq C(\mathrm{Co})^{3 / 2} K \sqrt{\tau} \sum_{n=2}^{N-1} E\left(u_{h}^{n+1}\right)\left\|\delta \delta u_{h}^{n+1}\right\|+C \mathrm{Co}\|\nabla \beta\|_{\infty} \tau \sum_{n=2}^{N-1}\left\|\tilde{u}_{h}^{n+1}\right\|\left\|\delta \delta u_{h}^{n+1}\right\| \\
& \quad+C(\mathrm{Co})^{2} \sum_{n=2}^{N-1}\left\|\delta \delta u_{h}^{n+1}\right\|^{2}+C \sqrt{\tau} \mathrm{Co}\left(\frac{\sqrt{\mathrm{Co}}}{\sqrt{\mathrm{Pe}}}+\sqrt{\mathrm{Co} \gamma}+\sqrt{\tau}\right) \sum_{n=2}^{N-1}\left\|L^{n+1}\right\|_{h}\left\|\delta \delta u_{h}^{n+1}\right\| .
\end{aligned}
$$

To bound the remaining two terms we use (2.11) followed by Young's inequality:

$$
\tau\left(c\left(\delta u_{h}^{N}, u_{h}^{N}\right)-c\left(\delta u_{h}^{1}, u_{h}^{2}\right)\right) \leq C \operatorname{Co}\left(\left\|u_{h}^{N}\right\|^{2}+\left\|\tilde{u}_{h}^{N+1}\right\|^{2}\right)+C \operatorname{Co}\left(\left\|u_{h}^{0}\right\|^{2}+\left\|u_{h}^{1}\right\|^{2}+\left\|u_{h}^{2}\right\|^{2}\right) .
$$

Here we also used that $\delta u_{h}^{N}=\tilde{u}_{h}^{N+1}-u_{h}^{N}$. Hence, we arrive at

$$
\begin{aligned}
S_{1} \leq & C \operatorname{Co}\left(\left\|u_{h}^{N}\right\|^{2}+\left\|\tilde{u}_{h}^{N+1}\right\|^{2}\right)+C \operatorname{Co}\left(\left\|u_{h}^{0}\right\|^{2}+\left\|u_{h}^{1}\right\|^{2}+\left\|u_{h}^{2}\right\|^{2}\right) \\
& +C \operatorname{Co}^{2} \sum_{n=2}^{N-1} \tau E\left(u_{h}^{n+1}\right)^{2}+C(\mathrm{Co})^{2} \sum_{n=2}^{N-1}\left\|\delta \delta u_{h}^{n+1}\right\|^{2} \\
& +C\|\nabla \beta\|_{\infty}^{2} \tau^{2} \sum_{n=2}^{N-1}\left\|\tilde{u}_{h}^{n+1}\right\|^{2}+\tau \sum_{n=2}^{N-1}\left\|L^{n+1}\right\|_{h}^{2} .
\end{aligned}
$$

Here we used that $\left(\frac{\sqrt{\mathrm{Co}}}{\sqrt{\mathrm{Pe}}}+\sqrt{\mathrm{Co} \gamma}+\sqrt{\tau}\right)$ is bounded in this case. Finally, using (3.10) and the fact that Co is sufficiently small we obtain

$$
\begin{aligned}
& \frac{1}{8}\left(\left\|u_{h}^{N}\right\|^{2}+\left\|\tilde{u}_{h}^{N+1}\right\|^{2}\right)+\frac{1}{2}\|\| u_{h} \|^{2} \\
& \quad \leq\left(\left\|u_{h}^{0}\right\|^{2}+\left\|u_{h}^{1}\right\|^{2}+\left\|u_{h}^{2}\right\|^{2}\right)+C \tau\left(\tau\|\nabla \beta\|_{\infty}^{2}+c_{L}\right) \sum_{n=1}^{N-1}\left\|u_{h}^{n+1}\right\|^{2}+9 \tau \sum_{n=1}^{N-1}\left\|L^{n+1}\right\|_{h}^{2} .
\end{aligned}
$$

We can now use the discrete Gronwall inequality (2.23) to get (3.9). 


\subsection{The case $\mathrm{Pe}>1$ for $p \geq 1$ with $4 / 3$-CFL condition}

We will now prove a stability result in the high Peclet regime $\mathrm{Pe}>1$ that holds for any polynomial order under more stringent $4 / 3$-CFL condition. In fact, we will not need the stabilization term $s(\cdot, \cdot)$ to guarantee this. Instead we test with the convection operator applied to the second order increment of $u_{h}^{n+1}$. This results in a term with a factor $\tau^{2} / h^{2}$ that must scale as $\sqrt{\tau}$ in order for it to be controlled through Gronwall's inequality. Clearly under the $4 / 3$-CFL condition, setting $\tau=\mathrm{Co}_{4 / 3} h^{4 / 3}$ we have $\tau^{2} / h^{2}=\sqrt{\tau} \tau^{3 / 2} / h^{2}=\sqrt{\tau} \mathrm{Co}_{4 / 3}^{3 / 2}$ and the desired control is achieved. The result holds for the standard Galerkin method as well.

Theorem 3.3. Suppose that $T=N \tau$, Pe $>1$ and that $\max \left\{\mathrm{Co}, \mathrm{Co}_{4 / 3}\right\}$ is sufficiently small only depending on geometric constants of the mesh and $\gamma$. Let $p \geq 1$. For $\left\{u_{h}^{n}\right\}$ solving (3.1) we have the following bound:

$$
\left\|u_{h}^{N}\right\|^{2} \leq\left(\left\|u_{h}^{0}\right\|^{2}+\left\|u_{h}^{1}\right\|^{2}+\left\|u_{h}^{2}\right\|^{2}+9 \tau \sum_{n=1}^{N-1}\left\|L^{n+1}\right\|_{h}^{2}\right) M,
$$

where

$$
M=8\left(1+\left(\frac{c_{L}}{4}+8\right) T e^{\left(\frac{c_{L}}{4}+8\right) T}\right) .
$$

Proof. Using the previous proof we only have to bound $S_{1}$ in the case, $\mathrm{Pe} \geq 1$ and $p>1$. We will use (3.11) and in particular use the same estimate (3.12). We are left to bound $\tau \sum_{n=2}^{N-1} c\left(\delta \delta u_{h}^{n+1}, \tau D_{\tau} u_{h}^{n+1}\right)$. To this end, we set $\psi_{h}=C_{h} \delta \delta u_{h}^{n+1}$ and see that

$$
c\left(\delta \delta u_{h}^{n+1}, \tau D_{\tau} u_{h}^{n+1}\right)=\tau\left(D_{\tau} u_{h}^{n+1}, \psi_{h}\right)_{\Omega} .
$$

Using the definition of the method (3.1) it follows that

$$
\tau^{2}\left(D_{\tau} u_{h}^{n+1}, \psi_{h}\right)_{\Omega}=M_{1}+M_{2}+M_{3}+M_{4} .
$$

where

$$
\begin{array}{ll}
M_{1}:=-\tau^{2} c\left(\tilde{u}_{h}^{n+1}, \psi_{h}\right), & M_{2}:=-\tau^{2} \gamma s\left(\tilde{u}_{h}^{n+1}, \psi_{h}\right), \\
M_{3}:=-\tau^{2} a\left(u_{h}^{n+1}, \psi_{h}\right), & M_{4}:=\tau^{2} L^{n+1}\left(\psi_{h}\right) .
\end{array}
$$

We use again the definition of $C_{h}$ and the estimate (2.13) to obtain

$$
M_{1}=-\tau^{2}\left(C_{h} \tilde{u}_{h}^{n+1}, \psi_{h}\right)_{\Omega} \leq \tau^{2}\left\|C_{h} \tilde{u}_{h}^{n+1}\right\|\left\|\psi_{h}\right\| \leq C \sqrt{\tau}\left(\mathrm{Co}_{4 / 3}\right)^{3 / 2}\left\|\tilde{u}_{h}^{n+1}\right\|\left\|\delta \delta u_{h}^{n+1}\right\| .
$$

Using (3.2a) and (2.14) we have

$$
M_{2} \leq \tau^{2} \gamma\left|\tilde{u}_{h}^{n+1}\right|_{s}\left|\psi_{h}\right|_{s} \leq \tau^{3 / 2} \sqrt{\gamma}\left|\tilde{u}_{h}^{n+1}\right|_{s} \sqrt{\gamma \mathrm{Co}}\left\|\psi_{h}\right\| \leq \sqrt{\tau} \sqrt{\gamma}\left|\tilde{u}_{h}^{n+1}\right|_{s} \sqrt{\gamma} \mathrm{Co}^{3 / 2}\left\|\delta \delta u_{h}^{n+1}\right\| .
$$

Similarly, we bound $M_{3}$ if we use (3.2b)

$$
M_{3} \leq \tau^{2}\left\|\sqrt{\mu} \nabla u_{h}^{n+1}\right\|\left\|\sqrt{\mu} \nabla \psi_{h}\right\| \leq C \tau^{2}\left\|\sqrt{\mu} \nabla u_{h}^{n+1}\right\| \frac{\sqrt{\mu}}{h}\left\|\psi_{h}\right\| \leq C \sqrt{\tau}\left\|\sqrt{\mu} \nabla u_{h}^{n+1}\right\| \frac{\mathrm{Co}^{3 / 2}}{\sqrt{\mathrm{Pe}}}\left\|\delta \delta u_{h}^{n+1}\right\| .
$$

Finally, using (3.3)

$$
\begin{aligned}
M_{4} & \leq \tau^{2}\left\|L_{h}^{n+1}\right\|_{h} \sqrt{E\left(\psi_{h}\right)^{2}+\left\|\psi_{h}\right\|^{2}} \\
& \leq C \sqrt{\tau}\left\|L_{h}^{n+1}\right\|_{h}\left(\sqrt{\gamma \mathrm{Co}}+\frac{\sqrt{\mathrm{Co}}}{\sqrt{\mathrm{Pe}}}+\sqrt{\tau}\right) \tau\left\|\psi_{h}\right\| .
\end{aligned}
$$


Hence, applying (2.14) we get

$$
M_{4} \leq \tau^{2}\left\|L_{h}^{n+1}\right\|_{h} \sqrt{E\left(\psi_{h}\right)^{2}+\left\|\psi_{h}\right\|^{2}} \leq C \sqrt{\tau}\left\|L_{h}^{n+1}\right\|_{h} \mathrm{Co}\left\|\delta \delta u_{h}^{n+1}\right\|,
$$

where we use that $\left(\gamma \mathrm{Co}+\frac{\mathrm{Co}}{\mathrm{Pe}}+\sqrt{\tau}\right)$ is bounded.

Hence, after using Young's inequality, (3.12) and the fac that $\mathrm{Pe}>1$ we arrive at

$$
\begin{aligned}
S_{1} \leq & C \operatorname{Co}\left(\left\|u_{h}^{N}\right\|^{2}+\left\|\tilde{u}_{h}^{N+1}\right\|^{2}\right)+C \operatorname{Co}\left(\left\|u_{h}^{0}\right\|^{2}+\left\|u_{h}^{1}\right\|^{2}+\left\|u_{h}^{2}\right\|^{2}\right) \\
& +\operatorname{Co} \sum_{n=2}^{N-1} \tau E\left(u_{h}^{n+1}\right)^{2}+C\left(\mathrm{Co}^{2}+\left(\mathrm{Co}_{4 / 3}\right)^{3}\right) \sum_{n=2}^{N-1}\left\|\delta \delta u_{h}^{n+1}\right\|^{2} \\
& +\tau \sum_{n=2}^{N-1}\left\|u_{h}^{n+1}\right\|^{2}+\tau \sum_{n=2}^{N-1}\left\|L^{n+1}\right\|_{h}^{2} .
\end{aligned}
$$

We now use (3.10) and the estimates $S_{2}$ and $S_{3}$ from the previous theorem. In addition, we use that Co and $\mathrm{Co}_{4 / 3}$ is sufficiently small to obtain

$$
\frac{1}{8}\left(\left\|u_{h}^{N}\right\|^{2}+\left\|\tilde{u}_{h}^{N+1}\right\|^{2}\right)+\frac{1}{2}\|\| u_{h}\left\|^{2} \leq\left(\left\|u_{h}^{0}\right\|^{2}+\left\|u_{h}^{1}\right\|^{2}+\left\|u_{h}^{2}\right\|^{2}\right)+9 \tau \sum_{n=1}^{N-1}\right\| L^{n+1}\left\|_{h}^{2}+\left(\frac{c_{L}}{32}+1\right) \tau \sum_{n=1}^{N-1}\right\| u_{h}^{n+1} \|^{2}
$$

The estimate (3.15) now follows from Gronwalls inequality (2.23).

We notice that $\left\|u_{h}^{2}\right\|$ appear in the right-hand side of some of the estimates; see for example (3.15). However, we we can easily show (we omit the details) that if Co sufficiently small

$$
\left\|u_{h}^{2}\right\| \leq 2\left(\left\|u_{h}^{0}\right\|^{2}+\left\|u_{h}^{1}\right\|^{2}\right)+C \tau\left\|L^{2}\right\|_{h}^{2} .
$$

If we combine Theorems 3.2 and 3.3 with this last inequality we get.

Corollary 3.4. Let $T=N \tau$. Let $\left\{u_{h}^{n}\right\}$ solving (3.1) we have the following bounds:

If $\mathrm{Pe} \leq 1$ and for all $p \geq 1$, if Co is sufficiently small we have:

$$
\left\|u_{h}^{N}\right\|^{2} \leq\left(1+\frac{T c_{L}}{8} e^{\frac{T c_{L}}{8}}\right)\left(\left\|u_{h}^{0}\right\|^{2}+\left\|u_{h}^{1}\right\|^{2}+32 \tau \sum_{n=1}^{N-1}\left\|L^{n+1}\right\|_{h}^{2}\right) .
$$

If $\mathrm{Pe}>1, p=1, \gamma>0$ and Co sufficiently small we obtain:

$$
\left\|u_{h}^{N}\right\|^{2} \leq C\left(\left\|u_{h}^{0}\right\|^{2}+\left\|u_{h}^{1}\right\|^{2}+\tau \sum_{n=1}^{N-1}\left\|L^{n+1}\right\|_{h}^{2}\right) M,
$$

where

$$
M=8\left(1+c T\left(\tau\|\nabla \beta\|_{\infty}^{2}+c_{L}\right) e^{c T\left(\tau\|\nabla \beta\|_{\infty}^{2}+c_{L}\right)}\right) .
$$

If $\mathrm{Pe}>1, p \geq 1$, and $\max \left\{\mathrm{Co}, \mathrm{Co}_{4 / 3}\right\}$ is sufficiently small we get:

$$
\left\|u_{h}^{N}\right\|^{2} \leq C\left(\left\|u_{h}^{0}\right\|^{2}+\left\|u_{h}^{1}\right\|^{2}+\tau \sum_{n=1}^{N-1}\left\|L^{n+1}\right\|_{h}^{2}\right) M,
$$

where $M$ is given in (3.18). 
Corollary 3.5. Let $T=N \tau$. Let $\left\{u_{h}^{n}\right\}$ solving (3.1). If $\mathrm{Pe}>1, p=1, \gamma>0$ and Co sufficiently small we obtain:

$$
\left.\left\|u_{h}\right\|\right|^{2} \leq C\left(1+T\left(\tau\|\nabla \beta\|_{\infty}^{2}+c_{L}\right)\right)\left(\left\|u_{h}^{0}\right\|^{2}+\left\|u_{h}^{1}\right\|^{2}+\tau \sum_{n=1}^{N-1}\left\|L^{n+1}\right\|_{h}^{2}\right) M,
$$

and $M$ is given in (3.18).

\section{A PRIORI ERror estimate For BDF2-IMEX IN The CASE Pe $>1$}

In this section we will study the error in the BDF2-IMEX method. We focus on the case Pe $>1$ for simplicity. If $u$ solves (2.2), its approximation is given by:

Find $u_{h}^{n+1} \in \stackrel{\circ}{V}_{h}$ such that for $n \geq 1$,

$$
\left(D_{\tau} u_{h}^{n+1}, v_{h}\right)_{\Omega}+c_{h}\left(\tilde{u}_{h}^{n+1}, v_{h}\right)+a\left(u_{h}^{n+1}, v_{h}\right)=\left(f, v_{h}\right)_{\Omega}, \quad \forall v_{h} \in \stackrel{\circ}{V}_{h},
$$

with $u_{h}^{i}=\pi_{h} u\left(\cdot, t_{i}\right), i=0,1$.

We can prove an error estimate in the case of $\mathrm{Pe}>1$ (i.e. convection dominated regime). This uses the stability of the time discretisation from the previous section applied to the perturbation equations, followed by consistency of the method and Lemma 2.1 to achieve the improved continuity of the convective term, that leads to the gain of $h^{\frac{1}{2}}$ in the error estimate compared to the standard Galerkin method.

Theorem 4.1. Let $u$ be the solution of (2.2) and $\left\{u_{h}^{n}\right\}_{n=0}^{N}$ be the solution of (4.1). Let $T=\tau N$ and assume that $\gamma>0$. Furthermore, suppose that $\mathrm{Co}$ is sufficiently small when $p=1$ and $\max \left\{\mathrm{Co}, \mathrm{Co}_{4 / 3}\right\}$ is sufficiently small when $p \geq 2$. Then,

$$
\left\|\pi_{h} u(T)-u_{h}^{N}\right\|^{2} \leq C\left(h^{2(p+1)}\left(\left\|u\left(t_{0}\right)\right\|_{H^{p+1}(\Omega)}^{2}+\left\|u\left(t_{1}\right)\right\|_{H^{p+1}(\Omega)}^{2}\right)+\mathrm{G}\right) \mathrm{M}
$$

where

$$
\begin{aligned}
\mathbf{G}:= & \left(\tau^{4}\left\|\partial_{t}^{3} u\right\|_{L^{2}\left([0, T], L^{2}(\Omega)\right)}^{2}+\|\beta\|_{\infty}^{2} \tau^{4}\left\|\partial_{t}^{2} u\right\|_{L^{2}\left([0, T], H^{1}(\Omega)\right)}^{2}\right) \\
& +\left(\mu h^{2 p}+\gamma\|\beta\|_{\infty} h^{2(p+1 / 2)}+\|\nabla \beta\|_{\infty}^{2} h^{2(p+1)}\right) \tau \sum_{j=1}^{N-1}\left\|u^{n+1}\right\|_{H^{p+1}(\Omega)}^{2}
\end{aligned}
$$

and

$$
\mathrm{M}=\left(1+c T\left(\tau\|\nabla \beta\|_{\infty}^{2}+1\right) e^{c T\left(\tau\|\nabla \beta\|_{\infty}^{2}+1\right)}\right) .
$$

Proof. Let $w_{h}^{n}=\pi_{h} u^{n}$ and let $e_{h}^{n}=w_{h}^{n}-u_{h}^{n}$ where $\pi_{h}$ is defined by (2.3). Moreover, we let $\eta_{h}^{n}=w_{h}^{n}-u^{n}$. Then, we have that

$$
\left(D_{\tau} e_{h}^{n+1}, v_{h}\right)_{\Omega}+c_{h}\left(\tilde{e}_{h}^{n+1}, v_{h}\right)+a\left(e_{h}^{n+1}, v_{h}\right)=L^{n+1}\left(v_{h}\right), \quad \forall v_{h} \in \stackrel{\circ}{V}_{h},
$$

where

$$
L^{n+1}\left(v_{h}\right):=\left(D_{\tau} w_{h}^{n+1}, v_{h}\right)_{\Omega}+c_{h}\left(\tilde{w}_{h}^{n+1}, v_{h}\right)+a\left(w_{h}^{n+1}, v_{h}\right)-\left(\left(\partial_{t} u^{n+1}, v_{h}\right)_{\Omega}+c\left(u^{n+1}, v_{h}\right)+a\left(u^{n+1}, v_{h}\right)\right) .
$$

We can write

$$
L^{n+1}\left(v_{h}\right):=\sum_{j=1}^{5} \Psi_{j}\left(v_{h}\right)
$$


where

$$
\begin{array}{ll}
\Psi_{1}\left(v_{h}\right):=\left(D_{\tau} u^{n+1}-\partial_{t} u^{n+1}, v_{h}\right)_{\Omega}, & \Psi_{2}\left(v_{h}\right):=c\left(\tilde{\eta}_{h}^{n+1}, v_{h}\right), \\
\Psi_{3}\left(v_{h}\right):=\gamma s\left(\tilde{\eta}_{h}^{n+1}, v_{h}\right), & \Psi_{4}\left(v_{h}\right):=a\left(\eta_{h}^{n+1}, v_{h}\right), \\
\Psi_{5}\left(v_{h}\right):=c\left(\tilde{u}^{n+1}-u^{n+1}, v_{h}\right) . &
\end{array}
$$

All the terms above can easily be bounded. However, we have to pay special care to $\Psi_{2}\left(v_{h}\right)$. Using the skewsymmetry of $c$, and the $L^{2}$-orthogonality of $\tilde{\eta}_{h}^{n+1}$ we can subtract an arbitrary $z_{h} \in \stackrel{\circ}{V_{h}}$ from the convective derivative. Then by the Cauchy-Schwarz inequality we see that

$$
\Psi_{2}\left(v_{h}\right)=-c\left(v_{h}, \tilde{\eta}_{h}^{n+1}\right)=-\left(\beta \cdot \nabla v_{h}-z_{h}, \tilde{\eta}_{h}^{n+1}\right)_{\Omega} \leq \inf _{z_{h} \in \dot{V}_{h}}\left\|\beta \cdot \nabla v_{h}-z_{h}\right\|\left\|\tilde{\eta}_{h}^{n+1}\right\| .
$$

Hence, using (2.9) we obtain

$$
\left|\Psi_{2}\left(v_{h}\right)\right| \leq C\left(\|\nabla \beta\|_{\infty}\left\|v_{h}\right\|+\frac{\sqrt{\|\beta\|_{\infty}}}{\sqrt{h} \sqrt{\gamma}} \sqrt{\gamma}\left|v_{h}\right|_{s}\right)\left\|\tilde{\eta}_{h}^{n+1}\right\| .
$$

As a consequence of this bound for $\Psi_{2}$ and by bounding all the other terms $\Psi_{i}$ using the Cauchy-Schwarz inequality we have

$$
\begin{aligned}
\left\|L^{n+1}\right\|_{h} \leq & \left\|D_{\tau} u^{n+1}-\partial_{t} u^{n+1}\right\|+\left\|\beta \cdot \nabla\left(\tilde{u}^{n+1}-u^{n+1}\right)\right\| \\
& +C\left(\|\nabla \beta\|_{\infty}+\frac{\sqrt{\|\beta\|_{\infty}}}{\sqrt{h} \sqrt{\gamma}}\right)\left\|\tilde{\eta}_{h}^{n+1}\right\|+C \sqrt{\gamma}\left|\tilde{\eta}_{h}^{n+1}\right|_{s}+C\left\|\sqrt{\mu} \nabla \eta_{h}^{n+1}\right\|_{L^{2}(\Omega)} .
\end{aligned}
$$

Using (2.19), and (2.20), the square of the first four terms of the right hand side can be bounded as follows.

$$
\begin{aligned}
\left\|D_{\tau} u^{n+1}-\partial_{t} u^{n+1}\right\|^{2} & \leq C \tau^{3} \int_{t_{n}}^{t_{n+1}}\left\|\partial_{t}^{3} u(\cdot, s)\right\|^{2} \mathrm{~d} s \\
\left\|\beta \cdot \nabla\left(\tilde{u}^{n+1}-u^{n+1}\right)\right\|^{2} & \leq C\|\beta\|_{\infty}^{2} \tau^{3}\left\|\partial_{t}^{2} \nabla u\right\|_{L^{2}\left(t_{n-1}, t_{n+1} ; L^{2}(\Omega)\right)}^{2}, \\
\left\|\tilde{\eta}_{h}^{n+1}\right\|^{2} & \leq C h^{2(p+1)}\left\|\tilde{u}^{n+1}\right\|_{H^{p+1}(\Omega)}^{2}, \\
\gamma\left|\tilde{\eta}_{h}^{n+1}\right|_{s}^{2} & \leq C \gamma\|\beta\|_{\infty} h^{2(p+1 / 2)}\left\|\tilde{u}^{n+1}\right\|_{H^{p+1}(\Omega)}^{2} \\
\left\|\sqrt{\mu} \nabla \eta_{h}^{n+1}\right\|_{L^{2}(\Omega)} & \leq \mu h^{2 p}\left\|u^{n+1}\right\|_{H^{p+1}(\Omega)}^{2}
\end{aligned}
$$

Therefore, combining the above inequalities we get

$$
\begin{aligned}
\tau \sum_{n=1}^{N-1}\left\|L^{n+1}\right\|_{h}^{2} \leq & C\left(\tau^{4}\left\|\partial_{t}^{3} u\right\|_{L^{2}\left([0, T], L^{2}(\Omega)\right)}^{2}+\|\beta\|_{\infty}^{2} \tau^{4}\left\|\partial_{t}^{2} u\right\|_{L^{2}\left([0, T], H^{1}(\Omega)\right)}^{2}\right) \\
& +C\left(\mu h^{2 p}+\gamma\|\beta\|_{\infty} h^{2(p+1 / 2)}+\|\nabla \beta\|_{\infty}^{2} h^{2(p+1)}\right) \tau \sum_{j=1}^{N-1}\left\|u^{n+1}\right\|_{H^{p+1}(\Omega)}^{2}
\end{aligned}
$$

The result now follows if we apply (3.16) and (3.17).

\subsection{Error estimate for the material derivative}

In this section we prove error estimates for the material derivative. We start with a lemma that shows that the projection of the material derivative of the error superconverges. The idea here is to test the perturbation equation with the projection of the material derivative and then control the residual terms using the error estimate of the previous section. 
Lemma 4.2. Let $u$ be the solution of (2.2) and $\left\{u_{h}^{n}\right\}_{n=0}^{N}$ be the solution of (4.1). Let $T=\tau N, p=1, \gamma>0$ and $\mathrm{Pe}>1$. Furthermore, suppose that $\mathrm{Co}$ is sufficiently small. If $m_{h}^{n+1}=D_{\tau} e_{h}^{n+1}+\beta \cdot \nabla \tilde{e}_{h}^{n+1}$ then

$$
\begin{aligned}
\tau \sum_{n=1}^{N-1} h\left\|\pi_{h} m_{h}^{n+1}\right\|^{2} \leq & C h\left((\gamma+1)\|\beta\|_{\infty}+1\right)\left(1+T\left(\tau\|\nabla \beta\|_{\infty}^{2}+1\right)\right) \\
& \times\left(h^{2(p+1)}\left(\left\|u\left(t_{0}\right)\right\|_{H^{p+1}(\Omega)}^{2}+\left\|u\left(t_{0}\right)\right\|_{H^{p+1}(\Omega)}^{2}\right)+\mathrm{G}\right) M .
\end{aligned}
$$

Proof. Using (4.3) we get

$$
h\left\|\pi_{h} m_{h}^{n+1}\right\|^{2}=h\left(m_{h}^{n+1}, \pi_{h} m_{h}^{n+1}\right)_{\Omega}=S_{1}+S_{2}+S_{3},
$$

where

$$
\begin{aligned}
& S_{1}=-h a\left(e_{h}^{n+1}, \pi_{h} m_{h}^{n+1}\right), \\
& S_{2}=-h \gamma s\left(e_{h}^{n+1}, \pi_{h} m_{h}^{n+1}\right), \\
& S_{3}=h L^{n+1}\left(\pi_{h} m_{h}^{n+1}\right) .
\end{aligned}
$$

We can easily show the following estimates

$$
\begin{aligned}
& S_{1} \leq \sqrt{\mu}\left\|\sqrt{\mu} \nabla e_{h}^{n+1}\right\|\left\|\pi_{h} m_{h}^{n+1}\right\|, \\
& S_{2} \leq C \sqrt{h} \sqrt{\gamma} \sqrt{\|\beta\|_{\infty}} \sqrt{\gamma}\left|e_{h}^{n+1}\right|_{s}\left\|\pi_{h} m_{h}^{n+1}\right\|, \\
& S_{3} \leq\left\|L^{n+1}\right\|_{h}\left(\sqrt{\mu}+\sqrt{h} \sqrt{\gamma} \sqrt{\|\beta\|_{\infty}}+h\right)\left\|\pi_{h} m_{h}^{n+1}\right\| .
\end{aligned}
$$

Thus, we get

$$
\begin{aligned}
h\left\|\pi_{h} m_{h}^{n+1}\right\|^{2} & \leq C\left(\mu+h \gamma\|\beta\|_{\infty}\right)\left(E\left(e_{h}^{n+1}\right)^{2}+\left\|L^{n+1}\right\|_{h}^{2}\right) \\
& \leq C h\left((\gamma+1)\|\beta\|_{\infty}+1\right)\left(E\left(e_{h}^{n+1}\right)^{2}+\left\|L^{n+1}\right\|_{h}^{2}\right)
\end{aligned}
$$

where we used that $\mathrm{Pe}>1$. This proves the following:

$$
\tau \sum_{n=1}^{N-1} h\left\|\pi_{h} m_{h}^{n+1}\right\|^{2} \leq C h\left((\gamma+1)\|\beta\|_{\infty}+1\right)\left(\left.\left\|e_{h}\right\|\right|^{2}+\tau \sum_{n=1}^{N-1}\left\|L^{n+1}\right\|_{h}^{2}\right) .
$$

We have shown (see (4.4)):

$$
\tau \sum_{n=1}^{N-1}\left\|L^{n+1}\right\|_{h}^{2} \leq C \mathrm{G}
$$

By using (3.20) we have

$$
\left\|\left|e_{h} \|\right|^{2} \leq C\left(1+T\left(\tau\|\nabla \beta\|_{\infty}^{2}+1\right)\right)\left(\left\|e_{h}^{0}\right\|^{2}+\left\|e_{h}^{1}\right\|^{2}+\tau \sum_{n=1}^{N-1}\left\|L^{n+1}\right\|_{h}^{2}\right) M .\right.
$$

Thus,

$$
\left\|\mid e_{h}\right\| \|^{2} \leq C\left(1+T\left(\tau\|\nabla \beta\|_{\infty}^{2}+1\right)\right)\left(h^{2(p+1)}\left(\left\|u\left(t_{0}\right)\right\|_{H^{p+1}(\Omega)}^{2}+\left\|u\left(t_{0}\right)\right\|_{H^{p+1}(\Omega)}^{2}\right)+\mathrm{G}\right) M .
$$


We can now prove an optimal estimate for the material derivative. This is achieved by observing that the stabilization controls the difference of the material derivative applied to the error and its projection on the finite element space, while the projection itself was shown to superconverge in the previous result.

Theorem 4.3. With the same hypothesis as in Lemma 4.2 the following estimate holds

$$
\begin{aligned}
\tau \sum_{n=1}^{N-1} h\left\|m_{h}^{n+1}\right\|^{2} \leq & C\left(T h\|\nabla \beta\|_{\infty}^{2}+\left(\|\beta\|_{\infty}+h\left((\gamma+1)\|\beta\|_{\infty}+1\right)\right)\left(1+T\left(\tau\|\nabla \beta\|_{\infty}^{2}+1\right)\right)\right) \\
& \times\left(h^{p+1}\left(\left\|u\left(t_{0}\right)\right\|_{H^{p+1}(\Omega)}^{2}+\left\|u\left(t_{1}\right)\right\|_{H^{p+1}(\Omega)}^{2}\right)+\mathrm{G}\right) \mathrm{M}
\end{aligned}
$$

where $\mathrm{G}$ and $\mathrm{M}$ are given in Theorem 4.1.

Proof. The triangle inequality gives

$$
\tau \sum_{n=1}^{N-1} h\left\|m_{h}^{n+1}\right\|^{2} \leq 2 \tau \sum_{n=1}^{N-1} h\left\|\pi_{h} m_{h}^{n+1}-m_{h}^{n+1}\right\|^{2}+2 \tau \sum_{n=1}^{N-1} h\left\|\pi_{h} m_{h}^{n+1}\right\|^{2} .
$$

Using (2.9) we have

$$
\begin{aligned}
\tau \sum_{n=1}^{N-1} h\left\|\pi_{h} m_{h}^{n+1}-m_{h}^{n+1}\right\|^{2} & \leq C\left(\|\nabla \beta\|_{\infty}^{2} h \tau \sum_{n=1}^{N-1}\left\|e_{h}^{n+1}\right\|^{2}+\tau\|\beta\|_{\infty} \sum_{n=1}^{N-1}\left|e_{h}^{n+1}\right|_{s}^{2}\right) \\
& \leq C\|\nabla \beta\|_{\infty}^{2} h \tau \sum_{n=1}^{N-1}\left\|e_{h}^{n+1}\right\|^{2}+C\|\beta\|_{\infty}\|\|_{h}\|\|^{2} .
\end{aligned}
$$

Applying (4.2) we get

$$
\tau \sum_{n=1}^{N-1}\left\|e_{h}^{n+1}\right\|^{2} \leq C T\left(h^{2(p+1)}\left(\left\|u\left(t_{0}\right)\right\|_{H^{2}(\Omega)}^{2}+\left\|u\left(t_{1}\right)\right\|_{H^{2}(\Omega)}^{2}\right)+\mathrm{G}\right) \mathrm{M}
$$

Therefore, also using (4.7) we obtain

$$
\begin{aligned}
& \tau \sum_{n=1}^{N-1} h\left\|\pi_{h} m_{h}^{n+1}-m_{h}^{n+1}\right\|^{2} \\
& \quad \leq C\left(T h\|\nabla \beta\|_{\infty}^{2}+\|\beta\|_{\infty}\left(1+T\left(\tau\|\nabla \beta\|_{\infty}^{2}+1\right)\right)\right)\left(h^{2(p+1)}\left(\left\|u\left(t_{0}\right)\right\|_{H^{2}(\Omega)}^{2}+\left\|u\left(t_{1}\right)\right\|_{H^{2}(\Omega)}^{2}\right)+\mathrm{G}\right) \mathrm{M} .
\end{aligned}
$$

Thus, combining these inequalities with (4.5) gives the result.

\section{Crank Nicolson imeX scheme}

In this section we will define the Crank Nicolson-IMEX method and prove that it is stable. The Crank Nicolson-IMEX method will read: Find $u_{h}^{n+1} \in \stackrel{\circ}{V}_{h}$ such that for $n \geq 1$,

$$
\left(\delta u_{h}^{n+1}, v_{h}\right)_{\Omega}+\tau c_{h}\left(\hat{u}_{h}^{n+1}, v_{h}\right)+\tau a\left(\bar{u}_{h}^{n+1}, v_{h}\right)=\tau L^{n+1}\left(v_{h}\right), \quad \forall v_{h} \in \stackrel{\circ}{V}_{h},
$$

where $u_{h}^{0}$ and $u_{h}^{1}$ are given. Here we use the notation

$$
\hat{u}_{h}^{n+1}:=\frac{3}{2} u_{h}^{n}-\frac{1}{2} u_{h}^{n-1}=\frac{\tilde{u}_{h}^{n+1}+u_{h}^{n}}{2}
$$




$$
\bar{u}_{h}^{n+1}:=\frac{u_{h}^{n+1}+u_{h}^{n}}{2} .
$$

We see that

$$
\hat{u}_{h}^{n+1}=\bar{u}_{h}^{n+1}-\frac{1}{2} \delta \delta u_{h}^{n+1}
$$

Here we define the triple norm as:

$$
\|v\| \|^{2}:=\sum_{n=1}^{N-1} \tau E\left(v^{n+1}\right)^{2} .
$$

In order to prove a stability result for the Crank-Nicoloson IMEX method, we need two different bounds for $\sum_{n=1}^{N-1}\left\|\delta \delta u_{h}^{n+1}\right\|^{2}$. One for the Pe $>1$ and one for $\mathrm{Pe} \leq 1$. The first is as follows.

Lemma 5.1. Let $u_{h}$ solve (5.1). If $\mathrm{Pe}>1$ and $\mathrm{Co}$ is sufficiently small, then the following estimate holds

$$
\sum_{n=1}^{N-1}\left\|\delta \delta u_{h}^{n+1}\right\|^{2} \leq C \operatorname{Co} \sum_{n=1}^{N-1}\left\|\delta u_{h}^{n+1}-P_{0}\left(\delta u_{h}^{n+1}\right)\right\|^{2}+C \tau \sum_{n=1}^{N-1}\left\|L^{n+1}\right\|_{h}^{2}+C \operatorname{Co}\left\|\bar{u}_{h}^{n+1}\right\| \|^{2} .
$$

Moreover, if $\mathrm{Pe}>1$ and $\max \left\{\mathrm{Co}_{4 / 3}, \mathrm{Co}\right\}$ is sufficiently small then we have the following estimate

$$
\sum_{n=1}^{N-1}\left\|\delta \delta u_{h}^{n+1}\right\|^{2} \leq C \tau \sum_{n=1}^{N-1}\left\|L^{n+1}\right\|_{h}^{2}+C \operatorname{Co}\left\|\bar{u}_{h}^{n+1} \mid\right\|^{2}+\tau \sum_{n=1}^{N-1}\left\|u_{h}^{n+1}\right\|^{2} .
$$

Proof. We see from (5.1) that

$$
\left(\delta \delta u_{h}^{n+1}, v_{h}\right)_{\Omega}+\tau c_{h}\left(\delta \hat{u}_{h}^{n+1}, v_{h}\right)+\tau a\left(\delta \bar{u}_{h}^{n+1}, v_{h}\right)=\tau \delta L^{n+1}\left(v_{h}\right), \quad \forall v_{h} \in \stackrel{\circ}{V}_{h} .
$$

Therefore, we easily have

$$
\sum_{n=1}^{N-1}\left\|\delta \delta u_{h}^{n+1}\right\|^{2}=S_{1}+S_{2}+S_{3}+S_{4}
$$

where

$$
\begin{aligned}
& S_{1}=-\tau \sum_{n=1}^{N-1} c\left(\delta \hat{u}_{h}^{n+1}, \delta \delta u_{h}^{n+1}\right) \\
& S_{2}=-\tau \sum_{n=1}^{N-1} \gamma s\left(\delta \hat{u}_{h}^{n+1}, \delta \delta u_{h}^{n+1}\right) \\
& S_{3}=-\tau \sum_{n=1}^{N-1} a\left(\delta \bar{u}_{h}^{n+1}, \delta \delta u_{h}^{n+1}\right) \\
& S_{4}=\tau \sum_{n=1}^{N-1} \delta L^{n+1}\left(\delta \delta u_{h}^{n+1}\right) .
\end{aligned}
$$

We start with an estimate of $S_{2}$. Using (5.2) and inverse estimates followed by Young's inequality we get:

$$
S_{2}=-\tau \sum_{n=1}^{N-1} \gamma s\left(\delta \bar{u}_{h}^{n+1}, \delta \delta u_{h}^{n+1}\right)+\frac{\tau}{2} \sum_{n=1}^{N-1} \gamma s\left(\delta \delta \delta u_{h}^{n+1}, \delta \delta u_{h}^{n+1}\right)
$$




$$
\leq\left(\gamma C \mathrm{Co}+\frac{1}{32}\right) \sum_{n=1}^{N-1}\left\|\delta \delta u_{h}^{n+1}\right\|^{2}+\operatorname{Co} \tau \sum_{n=1}^{N-1} \gamma\left|\bar{u}_{h}^{n+1}\right|_{s}^{2} .
$$

Similarly, we can show that

$$
S_{3} \leq \frac{1}{32} \sum_{n=1}^{N-1}\left\|\delta \delta u_{h}^{n+1}\right\|^{2}+C \frac{\mathrm{Co}}{\mathrm{Pe}} \tau \sum_{n=1}^{N-1}\left\|\sqrt{\mu} \nabla\left(\bar{u}_{h}^{n+1}\right)\right\|^{2} .
$$

Hence, by our assumption that $\mathrm{Pe}>1$ we get that

$$
S_{2}+S_{3} \leq\left(C \sqrt{\mathrm{Co}}+\frac{1}{16}\right) \sum_{n=1}^{N-1}\left\|\delta \delta u_{h}^{n+1}\right\|^{2}+C \mathrm{Co}\left\|\bar{u}_{h}^{n+1}\right\| \|^{2} .
$$

We can easily obtain using $(3.2 \mathrm{a})$ and $(3.2 \mathrm{~b})$

$$
S_{4} \leq \frac{1}{8} \sum_{n=1}^{N-1}\left\|\delta \delta u_{h}^{n+1}\right\|^{2}+C \tau\left(\frac{\sqrt{\mathrm{Co}}}{\sqrt{\mathrm{Pe}}}+\sqrt{\gamma \mathrm{Co}}+\sqrt{\tau}\right)^{2} \sum_{n=1}^{N-1}\left\|L^{n+1}\right\|_{h}^{2} .
$$

We now prove the estimate (5.3). We easily can show that using an inverse estimate that

$$
S_{1} \leq \frac{1}{8} \sum_{n=1}^{N-1}\left\|\delta \delta u_{h}^{n+1}\right\|^{2}+C \operatorname{Co} \sum_{n=1}^{N-1}\left\|\delta u_{h}^{n+1}-P_{0}\left(\delta u_{h}^{n+1}\right)\right\|^{2} .
$$

Thus, using that $\mathrm{Pe}>1$ and the fact that Co is sufficiently small we obtain:

$$
\sum_{n=1}^{N-1}\left\|\delta \delta u_{h}^{n+1}\right\|^{2} \leq C \operatorname{Co} \sum_{n=1}^{N-1}\left\|\delta u_{h}^{n+1}-P_{0}\left(\delta u_{h}^{n+1}\right)\right\|^{2}+C \tau \sum_{n=1}^{N-1}\left\|L^{n+1}\right\|_{h}^{2}+C \sqrt{\operatorname{Co}}\|\| \bar{u}_{h}^{n+1} \mid \|^{2} .
$$

This proves (5.3).

To prove (5.4) we write $S_{1}$.

$$
S_{1}=\tau \sum_{n=1}^{N-1} c\left(\delta \delta u_{h}^{n+1}, \delta \hat{u}_{h}^{n+1}\right)=\tau \sum_{n=1}^{N-1}\left(\psi_{h}^{n+1}, \delta \hat{u}_{h}^{n+1}\right)_{\Omega}=\frac{3 \tau}{2} \sum_{n=1}^{N-1}\left(\psi_{h}^{n+1}, \delta u_{h}^{n}\right)_{\Omega}-\frac{\tau}{2} \sum_{n=1}^{N-1}\left(\psi_{h}^{n+1}, \delta u_{h}^{n-1}\right)_{\Omega},
$$

where $\psi_{h}^{n}=C_{h}\left(\delta \delta u_{h}^{n}\right)$.

We estimate $\left(\psi_{h}^{n+1}, \delta u_{h}^{n}\right)_{\Omega}$. Using (5.1) we write

$$
\tau\left(\psi_{h}^{n+1}, \delta u_{h}^{n}\right)_{\Omega}=M_{1}+M_{2}+M_{3}+M_{4},
$$

where

$$
\begin{aligned}
& M_{1}:=-\tau^{2} c\left(\hat{u}_{h}^{n}, \psi_{h}^{n+1}\right), \\
& M_{2}:=-\tau^{2} \gamma s\left(\hat{u}_{h}^{n}, \psi_{h}^{n+1}\right), \\
& M_{3}:=-\tau^{2} a\left(\bar{u}_{h}^{n}, \psi_{h}^{n+1}\right), \\
& M_{4}:=\tau^{2} L^{n}\left(\psi_{h}^{n+1}\right) .
\end{aligned}
$$

Using inverse estimates and (2.13) we obtain

$$
M_{1} \leq C \sqrt{\tau}\left(\mathrm{Co}_{4 / 3}\right)^{3 / 2}\left\|\hat{u}_{h}^{n}\right\|\left\|\delta \delta u_{h}^{n+1}\right\|
$$




$$
\leq C\left(\mathrm{Co}_{4 / 3}\right)^{3 / 2} \tau\left\|\hat{u}_{h}^{n}\right\|^{2}+C\left(\mathrm{Co}_{4 / 3}\right)^{3 / 2}\left\|\delta \delta u_{h}^{n+1}\right\|^{2} .
$$

To estimate $M_{2}$, we use inverse estimates and (2.14) to obtain

$$
\begin{aligned}
M_{2} & =-\tau^{2} \gamma s\left(\bar{u}_{h}^{n}, \psi_{h}^{n+1}\right)+\frac{\tau^{2}}{2} \gamma s\left(\delta \delta u_{h}^{n}, \psi_{h}^{n+1}\right) \\
& \leq C \gamma \operatorname{Co}^{2}\left(\left\|\delta \delta u_{h}^{n}\right\|^{2}+\left\|\delta \delta u_{h}^{n+1}\right\|^{2}\right)+\tau \mathrm{Co}^{2} \gamma\left|\bar{u}_{h}^{n}\right|_{s}^{2} .
\end{aligned}
$$

Similarly, we get

$$
M_{3} \leq C \frac{\mathrm{Co}}{\sqrt{\mathrm{Pe}}}\left\|\delta \delta u_{h}^{n+1}\right\|^{2}+\tau \frac{\mathrm{Co}}{\sqrt{\mathrm{Pe}}}\left\|\sqrt{\mu} \nabla\left(\bar{u}_{h}^{n}\right)\right\|^{2} .
$$

Finally, again using inverse estimates and (2.14) to obtain

$$
M_{4} \leq C\left(\frac{\mathrm{Co}^{3}}{\mathrm{Pe}}+\gamma^{2} \mathrm{Co}^{3}+\mathrm{Co}^{2}\right)\left\|\delta \delta u_{h}^{n+1}\right\|^{2}+\frac{\tau}{2}\left\|L^{n}\right\|_{h}^{2} .
$$

Hence, using that $\mathrm{Pe}>1$ and that we can take $\mathrm{Co} \leq 1$ gives

$$
\begin{aligned}
\frac{3 \tau}{2} \sum_{n=1}^{N-1}\left(\psi_{h}^{n+1}, \delta u_{h}^{n}\right)_{\Omega} \leq & C\left(\left(\mathrm{Co}_{4 / 3}\right)^{3 / 2}+\mathrm{Co}+\mathrm{Co}^{3} \gamma^{2}\right) \sum_{n=1}^{N-1}\left\|\delta \delta u_{h}^{n+1}\right\|^{2}+\mathrm{Co}^{2}\left\|\bar{u}_{h}\right\| \|^{2} \\
& +\frac{\tau}{2} \sum_{n=1}^{N-1}\left\|L^{n+1}\right\|_{h}^{2}+C\left(\mathrm{Co}_{4 / 3}\right)^{3 / 2} \tau \sum_{n=1}^{N-1}\left\|u^{n+1}\right\|^{2}
\end{aligned}
$$

Similarly, we can prove the same estimate for $-\frac{\tau}{2} \sum_{n=1}^{N-1}\left(\psi_{h}^{n+1}, \delta u_{h}^{n-1}\right)_{\Omega}$ and so we get

$$
\begin{aligned}
S_{1} \leq & C\left(\left(\mathrm{Co}_{4 / 3}\right)^{3 / 2}+\mathrm{Co}+\mathrm{Co}^{3} \gamma^{2}\right) \sum_{n=1}^{N-1}\left\|\delta \delta u_{h}^{n+1}\right\|^{2}+\mathrm{Co}^{2}\left\|\bar{u}_{h}\right\| \|^{2} \\
& +\tau \sum_{n=1}^{N-1}\left\|L^{n+1}\right\|_{h}^{2}+C\left(\mathrm{Co}_{4 / 3}\right)^{3 / 2} \tau \sum_{n=1}^{N-1}\left\|u^{n+1}\right\|^{2} .
\end{aligned}
$$

If we combine the estimates of $S_{i}^{\prime} s$ and take $\max \left\{\mathrm{Co}_{4 / 3}, \mathrm{Co}\right\}$ we obtain (5.4).

The following alternative estimate will be useful when $\mathrm{Pe} \leq 1$.

Lemma 5.2. Assume that $\tau \leq 1$ and $\mathrm{Co}$ is sufficiently small. Let $u_{h}$ solve (5.1) then the following estimate holds

$$
\begin{aligned}
\sum_{n=1}^{N-1}\left\|\delta \delta u_{h}^{n+1}\right\|^{2} \leq & -\tau\left(\left\|\sqrt{\mu} \nabla\left(\delta u_{h}^{N}\right)\right\|^{2}-\left\|\sqrt{\mu} \nabla\left(\delta u_{h}^{1}\right)\right\|^{2}\right)+2 \sum_{n=0}^{N-1}\left\|\delta L^{n+1}\right\|_{h}^{2} \\
& +C \tau \operatorname{Co}(\operatorname{Pe}+1) \sum_{n=1}^{N-1} E\left(\bar{u}_{h}^{n+1}\right)^{2}+\tau^{2} \sum_{n=1}^{N-1}\left\|\sqrt{\mu} \nabla\left(\delta u_{h}^{n+1}\right)\right\|^{2}
\end{aligned}
$$

Proof.

$$
\sum_{n=1}^{N-1}\left\|\delta \delta u_{h}^{n+1}\right\|^{2}=S_{1}+S_{2}+S_{3}+S_{4}
$$


where $S_{i}^{\prime} s$ are given in (5.5).

We first notice that $a\left(\delta \bar{u}_{h}^{n+1}, \delta \delta u_{h}^{n+1}\right)=\frac{1}{2}\left(a\left(\delta u_{h}^{n+1}, \delta u_{h}^{n+1}\right)-a\left(\delta u_{h}^{n}, \delta u_{h}^{n}\right)\right)$. Hence,

$$
S_{3}=-\frac{\tau}{2}\left(\left\|\sqrt{\mu} \nabla\left(\delta u_{h}^{N}\right)\right\|^{2}-\left\|\sqrt{\mu} \nabla\left(\delta u_{h}^{1}\right)\right\|^{2}\right) .
$$

Using (5.2) we obtain

$$
S_{1}=-\tau \sum_{n=1}^{N-1} c\left(\delta \bar{u}_{h}^{n+1}, \delta \delta u_{h}^{n+1}\right)+\frac{\tau}{2} \sum_{n=1}^{N-1} c\left(\delta\left(\delta \delta u_{h}^{n+1}\right), \delta \delta u_{h}^{n+1}\right) .
$$

We then see that

$$
\begin{aligned}
-\tau \sum_{n=1}^{N-1} c\left(\delta \bar{u}_{h}^{n+1}, \delta \delta u_{h}^{n+1}\right) & \leq \frac{C \tau\|\beta\|_{\infty}}{\sqrt{\mu}} \sum_{n=1}^{N-1}\left\|\sqrt{\mu} \nabla\left(\delta \bar{u}_{h}^{n+1}\right)\right\|\left\|\delta \delta u_{h}^{n+1}\right\| \\
& \leq C \tau \operatorname{CoPe} \sum_{n=1}^{N-1}\left\|\sqrt{\mu} \nabla\left(\delta \bar{u}_{h}^{n+1}\right)\right\|^{2}+\frac{1}{16} \sum_{n=1}^{N-1}\left\|\delta \delta u_{h}^{n+1}\right\|^{2} .
\end{aligned}
$$

Similarly, now using inverse estimates, we get

$$
\begin{aligned}
\frac{\tau}{2} \sum_{n=1}^{N-1} c\left(\delta\left(\delta \delta u_{h}^{n+1}\right), \delta \delta u_{h}^{n+1}\right) & \leq C \operatorname{Co} \sum_{n=1}^{N-1}\left\|\delta\left(\delta \delta u_{h}^{n+1}\right)\right\|\left\|\delta \delta u_{h}^{n+1}\right\| \\
& \leq C \operatorname{Co} \sum_{n=1}^{N-1}\left\|\delta \delta u_{h}^{n+1}\right\|^{2}
\end{aligned}
$$

Hence, we have shown that

$$
S_{1} \leq C \tau \operatorname{CoPe} \sum_{n=1}^{N-1}\left\|\sqrt{\mu} \nabla\left(\delta \bar{u}_{h}^{n+1}\right)\right\|^{2}+\left(\frac{1}{16}+C \operatorname{Co}\right) \sum_{n=1}^{N-1}\left\|\delta \delta u_{h}^{n+1}\right\|^{2} .
$$

In a very similar fashion we can prove that

$$
S_{2} \leq C \tau \operatorname{Co} \sum_{n=1}^{N-1} \gamma\left|\delta \bar{u}_{h}^{n+1}\right|_{s}^{2}+\left(\frac{1}{16}+C \gamma \operatorname{Co}\right) \sum_{n=1}^{N-1}\left\|\delta \delta u_{h}^{n+1}\right\|^{2} .
$$

Therefore,

$$
S_{1}+S_{2} \leq\left(\frac{1}{8}+C(\gamma+1) \mathrm{Co}\right) \sum_{n=1}^{N-1}\left\|\delta \delta u_{h}^{n+1}\right\|^{2}+C \tau \mathrm{Co}(\mathrm{Pe}+1) \sum_{n=1}^{N-1} E\left(\bar{u}_{h}^{n+1}\right)^{2} .
$$

Here we used that $\sum_{n=1}^{N-1} E\left(\delta \bar{u}_{h}^{n+1}\right)^{2} \leq C \sum_{n=1}^{N-1} E\left(\bar{u}_{h}^{n+1}\right)^{2}$.

To bound $S_{4}$ we use the definition of the operator norm.

$$
\begin{aligned}
S_{4} & \leq \tau \sum_{n=1}^{N-1} \sqrt{E\left(\delta \delta u_{h}^{n+1}\right)^{2}+\left\|\delta \delta u_{h}^{n+1}\right\|^{2}}\left\|\delta L^{n+1}\right\|_{h} \\
& \leq \sum_{n=1}^{N-1}\left\|\delta L^{n+1}\right\|_{h}^{2}+\frac{\tau^{2}}{4} \sum_{n=1}^{N-1} E\left(\delta \delta u_{h}^{n+1}\right)^{2}+\frac{\tau^{2}}{4} \sum_{n=1}^{N-1}\left\|\delta \delta u_{h}^{n+1}\right\|^{2} .
\end{aligned}
$$


We can bound the energy as follows.

$$
\begin{aligned}
\frac{\tau^{2}}{4} \sum_{n=1}^{N-1} E\left(\delta \delta u_{h}^{n+1}\right)^{2} & =\frac{\tau^{2}}{4} \sum_{n=1}^{N-1}\left\|\sqrt{\mu} \nabla\left(\delta \delta u_{h}^{n+1}\right)\right\|^{2}+\frac{\tau^{2}}{4} \sum_{n=1}^{N-1} \gamma\left|\delta \delta u_{h}^{n+1}\right|_{s}^{2} \\
& \leq \tau^{2} \sum_{n=1}^{N-1}\left\|\sqrt{\mu} \nabla\left(\delta u_{h}^{n+1}\right)\right\|^{2}+C \operatorname{Co} \tau \sum_{n=1}^{N-1}\left\|\delta \delta u_{h}^{n+1}\right\|^{2}
\end{aligned}
$$

Hence,

$$
S_{4} \leq \sum_{n=1}^{N-1}\left\|\delta L^{n+1}\right\|_{h}^{2}+\tau^{2} \sum_{n=1}^{N-1}\left\|\sqrt{\mu} \nabla\left(\delta u_{h}^{n+1}\right)\right\|^{2}+\left(\frac{\tau^{2}}{4}+C \operatorname{Co} \tau\right) \sum_{n=1}^{N-1}\left\|\delta \delta u_{h}^{n+1}\right\|^{2} .
$$

We arrive at

$$
\begin{aligned}
\sum_{n=1}^{N-1}\left\|\delta \delta u_{h}^{n+1}\right\|^{2} \leq & -\frac{\tau}{2}\left(\left\|\sqrt{\mu} \nabla\left(\delta u_{h}^{N}\right)\right\|^{2}-\left\|\sqrt{\mu} \nabla\left(\delta u_{h}^{2}\right)\right\|^{2}\right)+\sum_{n=1}^{N-1}\left\|\delta L^{n+1}\right\|_{h}^{2} \\
& +C \tau \operatorname{Co}(\operatorname{Pe}+1) \sum_{n=1}^{N-1} E\left(\bar{u}_{h}^{n+1}\right)^{2}+\tau^{2} \sum_{n=1}^{N-1}\left\|\sqrt{\mu} \nabla\left(\delta u_{h}^{n+1}\right)\right\|^{2} \\
& +\left(\frac{\tau^{2}}{4}+\frac{1}{8}+C \operatorname{Co}(1+\tau)\right) \sum_{n=1}^{N-1}\left\|\delta \delta u_{h}^{n+1}\right\|^{2}
\end{aligned}
$$

The result follows by taking Co sufficiently small so that $\left(\frac{\tau^{2}}{4}+\frac{1}{8}+C \operatorname{Co}(1+\tau)\right) \leq \frac{1}{2}$.

In the case $p=1$ (and $\mathrm{Pe}>1$ ) we will need an auxiliarly result. This is the Crank-Nicolson equivalent to Lemma 3.1.

Lemma 5.3. Let $u_{h}$ solve (5.1).

If $p=1$ and $\gamma>0$ then the following estimate holds

$$
\begin{aligned}
\left\|\delta u_{h}^{n+1}-P_{0}\left(\delta u_{h}^{n+1}\right)\right\| \leq & C \sqrt{\tau \mathrm{Co}}\left(\frac{1}{\sqrt{\mathrm{Pe}}}+\sqrt{\gamma}+\frac{1}{\sqrt{\gamma}}\right) E\left(\bar{u}_{h}^{n+1}\right)+C \gamma \mathrm{Co}\left\|\delta \delta u_{h}^{n+1}\right\| \\
& +C \tau\|\nabla \beta\|_{\infty}\left\|\hat{u}_{h}^{n+1}\right\|+C \sqrt{\tau}\left(\frac{\sqrt{\mathrm{Co}}}{\sqrt{\mathrm{Pe}}}+\sqrt{\gamma \mathrm{Co}}+\sqrt{\tau}\right)\left\|L^{n+1}\right\|_{h} .
\end{aligned}
$$

If $p \geq 1$

$$
\begin{aligned}
\left\|\delta u_{h}^{n+1}\right\| \leq & C \sqrt{\tau \mathrm{Co}}\left(\frac{1}{\sqrt{\mathrm{Pe}}}+\sqrt{\gamma}+\frac{1}{\sqrt{\gamma}}\right) E\left(\bar{u}_{h}^{n+1}\right)+C \gamma \operatorname{Co}\left\|\delta \delta u_{h}^{n+1}\right\| \\
& +C \sqrt{\tau}\left(\frac{\sqrt{\mathrm{Co}}}{\sqrt{\mathrm{Pe}}}+\sqrt{\gamma \mathrm{Co}}+\sqrt{\tau}\right)\left\|L^{n+1}\right\|_{h}+C\|\beta\|_{\infty} \frac{\tau}{h}\left\|\hat{u}_{h}^{n+1}\right\| .
\end{aligned}
$$

Proof. We first prove (5.6). Let $y_{h}=\delta u_{h}^{n+1}$ and then we have by (5.1)

$$
\begin{aligned}
\left\|y_{h}-P_{0} y_{h}\right\|^{2} & =\left(y_{h}, y_{h}-P_{0}\left(y_{h}\right)\right)_{\Omega} \\
& =\left(y_{h}, \pi_{h}\left(y_{h}-P_{0}\left(y_{h}\right)\right)\right)_{\Omega} \\
& =-\tau c_{h}\left(\hat{u}_{h}^{n+1}, \pi_{h}\left(y_{h}-P_{0}\left(y_{h}\right)\right)\right)-\tau a\left(\bar{u}_{h}^{n+1}, \pi_{h}\left(y_{h}-P_{0}\left(y_{h}\right)\right)\right)
\end{aligned}
$$




$$
+\tau L^{n+1}\left(\pi_{h}\left(y_{h}-P_{0}\left(y_{h}\right)\right)\right) .
$$

We use the Cauchy-Schwarz inequality followed inverse estimates to bound the symmetric terms

$$
\begin{aligned}
-\tau a\left(\bar{u}_{h}^{n+1}, \pi_{h}\left(y_{h}-P_{0}\left(y_{h}\right)\right)\right) & \leq C \frac{\sqrt{\mu} \tau}{h}\left\|\sqrt{\mu} \nabla \bar{u}_{h}^{n+1}\right\|\left\|y_{h}-P_{0}\left(y_{h}\right)\right\| \\
& \leq C \frac{\sqrt{\tau \mathrm{Co}}}{\sqrt{\mathrm{Pe}}}\left\|\sqrt{\mu} \nabla \bar{u}_{h}^{n+1}\right\|\left\|y_{h}-P_{0}\left(y_{h}\right)\right\|,
\end{aligned}
$$

and for the stabilization we apply $(2.12)$

$$
\begin{aligned}
-\tau \gamma s\left(\hat{u}_{h}^{n+1}, \pi_{h}\left(y_{h}-P_{0}\left(y_{h}\right)\right)\right) & \leq C \sqrt{\tau} \sqrt{\gamma \operatorname{Co}} \sqrt{\gamma}\left|\hat{u}_{h}^{n+1}\right|_{s}\left\|y_{h}-P_{0}\left(y_{h}\right)\right\| \\
& \leq C\left(\sqrt{\tau} \sqrt{\gamma \operatorname{Co}} \sqrt{\gamma}\left|\bar{u}_{h}^{n+1}\right|_{s}+\gamma \operatorname{Co}\left\|\delta \delta u_{h}^{n+1}\right\|\right)\left\|y_{h}-P_{0}\left(y_{h}\right)\right\| .
\end{aligned}
$$

Next we bound $\tau L^{n+1}\left(\pi_{h}\left(y_{h}-P_{0}\left(y_{h}\right)\right)\right)$ using (3.4) and (3.3).

$$
\begin{aligned}
\tau L^{n+1}\left(\pi_{h}\left(y_{h}-P_{0}\left(y_{h}\right)\right)\right) & \leq \tau\left\|L^{n+1}\right\|_{h} \sqrt{E\left(\pi_{h}\left(y_{h}-P_{0}\left(y_{h}\right)\right)\right)^{2}+\left\|\pi_{h}\left(y_{h}-P_{0}\left(y_{h}\right)\right)\right\|^{2}} \\
& \leq C \sqrt{\tau}\left(\frac{\sqrt{\mathrm{Co}}}{\sqrt{\mathrm{Pe}}}+\sqrt{\gamma \mathrm{Co}}+\sqrt{\tau}\right)\left\|L^{n+1}\right\|_{h}\left\|y_{h}-P_{0}\left(y_{h}\right)\right\| .
\end{aligned}
$$

It only remains to bound the contribution from the form $c$.

$$
\begin{aligned}
-\tau c\left(\hat{u}_{h}^{n+1}, \pi_{h}\left(y_{h}-P_{0}\left(y_{h}\right)\right)\right)= & -\tau\left(\left(\beta-\beta_{0}\right) \cdot \nabla \hat{u}_{h}^{n+1}, \pi_{h}\left(y_{h}-P_{0}\left(y_{h}\right)\right)\right)_{\Omega} \\
& -\tau\left(\beta_{0} \cdot \nabla \hat{u}_{h}^{n+1}-w_{h}^{n+1},\left(I-\pi_{h}\right)\left(y_{h}-P_{0}\left(y_{h}\right)\right)\right)_{\Omega} .
\end{aligned}
$$

Here $w_{h} \in \stackrel{\circ}{V}_{h}$ is arbitrary. Note that we crucially used that $p=1$ which implies that $\beta_{0} \cdot \nabla \hat{u}_{h}^{n+1} \in W_{h}$.

Hence, using (2.6) and (2.7) we obtain

$$
\begin{aligned}
-\tau c\left(\hat{u}_{h}^{n+1}, \pi_{h}\left(y_{h}-P_{0}\left(y_{h}\right)\right)\right) & \leq C \tau\left(\|\nabla \beta\|_{\infty}\left\|\hat{u}_{h}^{n+1}\right\|+\|\beta\|_{\infty}^{1 / 2} h^{-1 / 2}\left|\hat{u}_{h}^{n+1}\right|_{s}\right)\left\|y_{h}-P_{0}\left(y_{h}\right)\right\| \\
& \leq C \sqrt{\tau}\left(\sqrt{\tau}\|\nabla \beta\|_{\infty}\left\|\hat{u}_{h}^{n+1}\right\|+\sqrt{\mathrm{Co}} \frac{1}{\sqrt{\gamma}} \sqrt{\gamma}\left|\hat{u}_{h}^{n+1}\right|_{s}\right)\left\|y_{h}-P_{0}\left(y_{h}\right)\right\| .
\end{aligned}
$$

Finally, by the triangle inequality and (2.12) we have the bound $\left|\hat{u}_{h}^{n+1}\right|_{s} \leq\left|\bar{u}_{h}^{n+1}\right|_{s}+\frac{C \sqrt{\mathrm{Co}}}{\sqrt{\tau}}\left\|\delta \delta u_{h}^{n+1}\right\|$. Combining the above inequalities gives (5.6).

Now we prove (5.7). Using (5.1) we have

$$
\left\|y_{h}\right\|^{2}=-\tau c\left(\hat{u}_{h}^{n+1}, y_{h}\right)-\tau \gamma s\left(\hat{u}_{h}^{n+1}, y_{h}\right)-\tau a\left(\bar{u}_{h}^{n+1}, y_{h}\right)+\tau L^{n+1}\left(y_{h}\right) .
$$

Similar to what we did above we can show that

$$
\begin{aligned}
-\tau \gamma s\left(\hat{u}_{h}^{n+1}, y_{h}\right)-\tau a\left(\bar{u}_{h}^{n+1}, y_{h}\right)+\tau L^{n+1}\left(y_{h}\right) \leq & C \frac{\sqrt{\tau \mathrm{Co}}}{\sqrt{\mathrm{Pe}}}\left\|\sqrt{\mu} \nabla \bar{u}_{h}^{n+1}\right\|\left\|y_{h}\right\| \\
& +C\left(\sqrt{\tau} \sqrt{\gamma \mathrm{Co}} \sqrt{\gamma}\left|\bar{u}_{h}^{n+1}\right|_{s}+\gamma \operatorname{Co}\left\|\delta \delta u_{h}^{n+1}\right\|\right)\left\|y_{h}\right\| \\
& +C \sqrt{\tau}\left(\frac{\sqrt{\mathrm{Co}}}{\sqrt{\mathrm{Pe}}}+\sqrt{\gamma \mathrm{Co}}+\sqrt{\tau}\right)\left\|L^{n+1}\right\|_{h}\left\|y_{h}\right\| .
\end{aligned}
$$

We then bound the remaining term using inverse estimates

$$
-\tau c\left(\hat{u}_{h}^{n+1}, y_{h}\right) \leq \frac{C \tau}{h}\|\beta\|_{\infty}\left\|\hat{u}_{h}^{n+1}\right\|\left\|y_{h}\right\| .
$$

Combining the above estimates proves (5.7). 
Corollary 5.4. Let $u_{h}$ solve (5.1).

Let $p=1, \mathrm{Pe}>1$ and $\gamma>0$. If Co is sufficiently small the following estimate holds

$$
\sum_{n=1}^{N-1}\left\|\delta \delta u_{h}^{n+1}\right\|^{2}+\sum_{n=1}^{N-1}\left\|\delta u_{h}^{n+1}-P_{0}\left(\delta u_{h}^{n+1}\right)\right\|^{2} \leq C \mathrm{Co}\|\| \bar{u}_{h}^{n+1} \mid\left\|^{2}+C \tau^{2}\right\| \nabla \beta\left\|_{\infty}^{2} \sum_{n=0}^{N-1}\right\| u_{h}^{n+1}\left\|^{2}+C \tau \sum_{n=0}^{N-1}\right\| L^{n+1} \|_{h}^{2} .
$$

Let $p \geq 1$ and $\mathrm{Pe}>1$. If $\max \left\{\mathrm{Co}, \mathrm{Co}_{4 / 3}\right\}$ is sufficiently small the following estimate holds

$$
\sum_{n=1}^{N-1}\left\|\delta u_{h}^{n+1}\right\|^{2} \leq C \mathrm{Co}\|\| \bar{u}_{h}\|\|^{2}+C \tau \sum_{n=1}^{N-1}\left\|L^{n+1}\right\|_{h}^{2}+C\left(\sqrt{\tau}\left(\mathrm{Co}_{4 / 3}\right)^{2}+\tau\right) \sum_{n=1}^{N-1}\left\|u_{h}^{n+1}\right\|^{2} .
$$

Proof. We first prove (5.9). From (5.6) we get

$$
\sum_{n=1}^{N-1}\left\|\delta u_{h}^{n+1}-P_{0}\left(\delta u_{h}^{n+1}\right)\right\|^{2} \leq C \mathrm{Co}\left\|\bar{u}_{h}\right\|^{2}+C \tau^{2}\|\nabla \beta\|_{\infty}^{2} \sum_{n=1}^{N-1}\left\|u_{h}^{n+1}\right\|^{2}+C \tau \sum_{n=1}^{N-1}\left\|L^{n+1}\right\|_{h}^{2}+C \sum_{n=0}^{N-1}\left\|\delta \delta u_{h}^{n+1}\right\|^{2} .
$$

Using (5.3) we thus obtain

$$
\begin{aligned}
\sum_{n=1}^{N-1}\left\|\delta u_{h}^{n+1}-P_{0}\left(\delta u_{h}^{n+1}\right)\right\|^{2} \leq & C \mathrm{Co}\left\|\bar{u}_{h}^{n+1} \mid\right\|^{2}+C \tau^{2}\|\nabla \beta\|_{\infty}^{2} \sum_{n=1}^{N-1}\left\|u_{h}^{n+1}\right\|^{2} \\
& +C \tau \sum_{n=1}^{N-1}\left\|L^{n+1}\right\|_{h}^{2}+C \operatorname{Co} \sum_{n=0}^{N-1}\left\|\delta u_{h}^{n+1}-P_{0}\left(\delta u_{h}^{n+1}\right)\right\|^{2} .
\end{aligned}
$$

If Co is sufficiently small we get

$$
\sum_{n=1}^{N-1}\left\|\delta u_{h}^{n+1}-P_{0}\left(\delta u_{h}^{n+1}\right)\right\|^{2} \leq C \mathrm{Co}\left\|\bar{u}_{h}^{n+1} \mid\right\|^{2}+C \tau^{2}\|\nabla \beta\|_{\infty}^{2} \sum_{n=1}^{N-1}\left\|u_{h}^{n+1}\right\|^{2}+C \tau \sum_{n=1}^{N-1}\left\|L^{n+1}\right\|_{h}^{2} .
$$

The bound for $\sum_{n=1}^{N-1}\left\|\delta \delta u_{h}^{n+1}\right\|^{2}$ follows from this and using (5.3) again.

Now we prove (5.10). From (5.7) we get

$$
\sum_{n=1}^{N-1}\left\|\delta u_{h}^{n+1}\right\|^{2} \leq C \mathrm{Co}\left\|\bar{u}_{h}\right\|^{2}+C \tau \sum_{n=1}^{N-1}\left\|L^{n+1}\right\|_{h}^{2}+C \sum_{n=0}^{N-1}\left\|\delta \delta u_{h}^{n+1}\right\|^{2}+\frac{C \tau^{2}\|\beta\|_{\infty}^{2}}{h^{2}} \sum_{n=1}^{N-1}\left\|u_{h}^{n+1}\right\|^{2} .
$$

The estimate (5.10) now follows from (5.4) and using the definiton of $\mathrm{Co}_{4 / 3}$.

We can now proceed and prove stability results for the extrapolated Crank-Nicolson scheme. The main ideas used are similar to the BDF2 case, but requires careful adaption to the Crank-Nicolson scheme, since this scheme does not have the same favorable dissipation properties as the BDF2 scheme.

Theorem 5.5. Let $T=N \tau$. Suppose that Co is chosen sufficiently small only depending on geometric constants of the mesh and $\gamma$. For $\left\{u_{h}^{n}\right\}$ solving (5.1) we have the following bounds:

If $\mathrm{Pe} \leq 1$ then for all $p \geq 1$,

$$
\left\|u_{h}^{N}\right\|^{2}+\tau\left\|\sqrt{\mu} \nabla\left(\delta u_{h}^{N}\right)\right\|^{2} \leq\left(1+T e^{T}\right) M,
$$


where

$$
M=\tau\left\|\sqrt{\mu} \nabla\left(\delta u_{h}^{1}\right)\right\|^{2}+\left\|u_{h}^{1}\right\|^{2}+16 \tau \sum_{n=1}^{N-1}\left\|L^{n+1}\right\|_{h}^{2}+4 \sum_{n=1}^{N-1}\left\|\delta L^{n+1}\right\|_{h}^{2} .
$$

If $\mathrm{Pe}>1$ and $p=1, \gamma>0$ then

$$
\left\|u_{h}^{N}\right\|^{2} \leq\left(2\left\|u_{h}^{1}\right\|^{2}+\left\|u_{h}^{2}\right\|^{2}+36 \tau \sum_{n=1}^{N-1}\left\|L^{n+1}\right\|_{h}^{2}\right) M
$$

where

$$
M=\left(1+T\left(\tau\|\nabla \beta\|_{\infty}^{2}+\frac{c_{L}}{8}\right) e^{T\left(\tau\|\nabla \beta\|_{\infty}^{2}+\frac{c_{L}}{8}\right)}\right) .
$$

If $\mathrm{Pe}>1$ and $p \geq 1$, and $\max \left\{\mathrm{Co}, \mathrm{Co}_{4 / 3}\right\}$ is sufficiently small we have

$$
\left\|u_{h}^{N}\right\|^{2} \leq\left(2\left\|u_{h}^{1}\right\|^{2}+\left\|u_{h}^{2}\right\|^{2}+36 \tau \sum_{n=1}^{N-1}\left\|L^{n+1}\right\|_{h}^{2}\right) M
$$

with $M$ as in (5.13).

Proof. Choose $v_{h}=\bar{u}_{h}^{n+1}$ in (5.1) and use (5.2), (2.10) to get

$$
\frac{1}{2}\left\|u_{h}^{n+1}\right\|^{2}-\frac{1}{2}\left\|u_{h}^{n}\right\|^{2}+\tau E\left(\bar{u}_{h}^{n+1}\right)^{2}=\frac{\tau}{2} c_{h}\left(\delta \delta u_{h}^{n+1}, \bar{u}_{h}^{n+1}\right)+\tau L^{n+1}\left(\bar{u}_{h}^{n+1}\right) .
$$

Taking the sum from $1 \leq n \leq N-1$ in (5.15) we arrive at

$$
\frac{1}{2}\left\|u_{h}^{N}\right\|^{2}-\frac{1}{2}\left\|u_{h}^{1}\right\|^{2}+\left.\left\|\bar{u}_{h}\right\|\right|^{2}=S_{1}+S_{2}+S_{3},
$$

where

$$
\begin{aligned}
S_{1} & :=\frac{\tau}{2} \sum_{n=1}^{N-1} c\left(\delta \delta u_{h}^{n+1}, \bar{u}_{h}^{n+1}\right) \\
S_{2} & :=\frac{\tau}{2} \sum_{n=1}^{N-1} \gamma s\left(\delta \delta u_{h}^{n+1}, \bar{u}_{h}^{n+1}\right) \\
S_{3} & :=\tau \sum_{n=1}^{N-1} L^{n+1}\left(\bar{u}_{h}^{n+1}\right) .
\end{aligned}
$$

Let us estimate $S_{3}$. We have

$$
\begin{aligned}
S_{3} & \leq \tau \sum_{n=1}^{N-1}\left\|L^{n+1}\right\|_{h} \sqrt{E\left(\bar{u}_{h}^{n+1}\right)^{2}+\left\|\bar{u}_{h}^{n+1}\right\|^{2}} \\
& \leq 8 \tau \sum_{n=1}^{N-1}\left\|L^{n+1}\right\|_{h}^{2}+\left.\frac{1}{32}\left\|\bar{u}_{h}\right\|\right|^{2}+\frac{c_{L}}{32} \tau \sum_{n=1}^{N-1}\left\|u_{h}^{n+1}\right\|^{2} .
\end{aligned}
$$

We now consider three cases. 
Case 1. $\mathrm{Pe}<1, p \geq 1$.

In this case, we can easily show that

$$
\begin{aligned}
S_{2} & =-\frac{\tau}{2} \sum_{n=1}^{N-1} c\left(\bar{u}_{h}^{n+1}, \delta \delta u_{h}^{n+1}\right) \\
& \leq \frac{\|\beta\|_{\infty} \tau}{\sqrt{\mu}} \sum_{n=1}^{N-1}\left\|\sqrt{\mu} \nabla\left(\bar{u}_{h}^{n+1}\right)\right\|\left\|\delta \delta u_{h}^{n+1}\right\| \\
& \leq \sqrt{\operatorname{CoPe}} \sqrt{\tau} \sum_{n=1}^{N-1}\left\|\sqrt{\mu} \nabla\left(\bar{u}_{h}^{n+1}\right)\right\|\left\|\delta \delta u_{h}^{n+1}\right\| \\
& \leq \tau C \operatorname{Co} \sum_{n=1}^{N-1}\left\|\sqrt{\mu} \nabla\left(\bar{u}_{h}^{n+1}\right)\right\|^{2}+\frac{1}{4} \sum_{n=1}^{N-1}\left\|\delta \delta u_{h}^{n+1}\right\|^{2} .
\end{aligned}
$$

Similarly,

$$
\begin{aligned}
S_{1} & =C \sqrt{\operatorname{Co}} \sqrt{\tau} \sum_{n=1}^{N-1} \gamma\left|\bar{u}_{h}^{n+1}\right|_{s}\left\|\delta \delta u_{h}^{n+1}\right\| \\
& \leq C \operatorname{Co} \gamma \tau \sum_{n=1}^{N-1} \gamma\left|\bar{u}_{h}^{n+1}\right|_{s}^{2}+\frac{1}{4} \sum_{n=1}^{N-1}\left\|\delta \delta u_{h}^{n+1}\right\|^{2}
\end{aligned}
$$

Using (5.16) and combining the above inequalities we get

$$
\begin{aligned}
\frac{1}{2}\left\|u_{h}^{N}\right\|^{2}-\frac{1}{2}\left\|u_{h}^{1}\right\|^{2}+\left.\left\|\bar{u}_{h}\right\|\right|^{2} \leq & \left.\left(\frac{1}{32}+\operatorname{Co}(1+\gamma)\right)\left\|\bar{u}_{h}\right\|\right|^{2}+8 \tau \sum_{n=1}^{N-1}\left\|L^{n+1}\right\|_{h}^{2} \\
& +\frac{c_{L}}{32} \tau \sum_{n=1}^{N-1}\left\|u_{h}^{n+1}\right\|^{2}+\frac{1}{2} \sum_{n=1}^{N-1}\left\|\delta \delta u_{h}^{n+1}\right\|^{2}
\end{aligned}
$$

If we use Lemma 5.2 and the fact that $\mathrm{Pe} \leq 1$ we get

$$
\begin{aligned}
\frac{1}{2}\left\|u_{h}^{N}\right\|^{2}+\frac{\tau}{2}\left\|\sqrt{\mu} \nabla\left(\delta u_{h}^{N}\right)\right\|^{2}+\left.\left\|\bar{u}_{h}\right\|\right|^{2} \leq & \frac{\tau}{2}\left\|\sqrt{\mu} \nabla\left(\delta u_{h}^{1}\right)\right\|^{2}+\frac{1}{2}\left\|u_{h}^{1}\right\|^{2}+8 \tau \sum_{n=0}^{N-1}\left\|L^{n+1}\right\|_{h}^{2} \\
& +2 \sum_{n=1}^{N-1}\left\|\delta L^{n+1}\right\|_{h}^{2}+\tau^{2} \sum_{n=0}^{N-1}\left\|\sqrt{\mu} \nabla\left(\delta u_{h}^{n+1}\right)\right\|^{2} \\
& +\frac{c_{L}}{32} \tau \sum_{n=1}^{N-1}\left\|u_{h}^{n+1}\right\|^{2}+\left(\frac{1}{32}+C \operatorname{Co}(1+\gamma)\right)\left\|\bar{u}_{h}\right\| \|^{2}
\end{aligned}
$$

Choosing Co sufficiently small gives

$$
\begin{aligned}
\frac{1}{2}\left\|u_{h}^{N}\right\|^{2}+\frac{\tau}{2}\left\|\sqrt{\mu} \nabla\left(\delta u_{h}^{N}\right)\right\|^{2}+\frac{1}{2}\left\|\mid \bar{u}_{h}\right\| \|^{2} \leq & \frac{\tau}{2}\left\|\sqrt{\mu} \nabla\left(\delta u_{h}^{2}\right)\right\|^{2}+\frac{1}{2}\left\|u_{h}^{1}\right\|^{2}+8 \tau \sum_{n=0}^{N-1}\left\|L^{n+1}\right\|_{h}^{2} \\
& +2 \sum_{n=1}^{N-1}\left\|\delta L^{n+1}\right\|_{h}^{2}+\tau^{2} \sum_{n=1}^{N-1}\left\|\sqrt{\mu} \nabla\left(\delta u_{h}^{n+1}\right)\right\|^{2}
\end{aligned}
$$




$$
+\frac{c_{L}}{32} \tau \sum_{n=1}^{N-1}\left\|u_{h}^{n+1}\right\|^{2}
$$

Applying Gronwall's inequality gives (5.11).

Case 2. $\mathrm{Pe}>1, p=1, \gamma>0$.

Now we estimate $S_{2}$. Using the arithmetic-geometric mean inequality and inverse estimates we obtain

$$
\begin{aligned}
S_{2} & \leq C \tau \sum_{n=1}^{N-1} \gamma \frac{\sqrt{\|\beta\|_{\infty}}}{\sqrt{h}}\left|\bar{u}_{h}^{n+1}\right|_{s}\left\|\delta \delta u_{h}^{n+1}\right\| \\
& \leq \frac{1}{32}\left\|\bar{u}_{h}\right\|\left\|^{2}+C \gamma \operatorname{Co} \sum_{n=1}^{N-1}\right\| \delta \delta u_{h}^{n+1} \|^{2} .
\end{aligned}
$$

To estimate $S_{1}$ we re-write it as follows

$$
S_{1}=D_{1}+D_{2}
$$

where

$$
\begin{aligned}
& D_{1}:=\frac{\tau}{2} \sum_{n=1}^{N-1} c\left(\delta \delta u_{h}^{n+1}, u_{h}^{n+1}\right), \\
& D_{2}:=-\frac{\tau}{2} \sum_{n=1}^{N-1} c\left(\delta \delta u_{h}^{n+1}, \delta u_{h}^{n+1}\right) .
\end{aligned}
$$

We use integration by parts and inverse estimates to obtain

$$
\begin{aligned}
D_{2} & =\frac{\tau}{2} \sum_{n=1}^{N-1} c\left(\delta u_{h}^{n+1}, \delta \delta u_{h}^{n+1}\right) \\
& \leq C \operatorname{Co} \sum_{n=1}^{N-1}\left\|\delta u_{h}^{n+1}-P_{0}\left(\delta u_{h}^{n+1}\right)\right\|\left\|\delta \delta u_{h}^{n+1}\right\| \\
& \leq C \operatorname{Co} \sum_{n=1}^{N-1}\left\|\delta u_{h}^{n+1}-P_{0}\left(\delta u_{h}^{n+1}\right)\right\|^{2}+C \operatorname{Co} \sum_{n=1}^{N-1}\left\|\delta \delta u_{h}^{n+1}\right\|^{2} .
\end{aligned}
$$

To estimate $D_{1}$ we use summation by parts (2.22) to write

$$
\begin{aligned}
D_{1} & =-\frac{\tau}{2} \sum_{n=2}^{N-1} c\left(\delta u_{h}^{n}, \delta u_{h}^{n+1}\right)+\frac{\tau}{2}\left(c\left(\delta u_{h}^{N}, u_{h}^{N}\right)-c\left(\delta u_{h}^{1}, u_{h}^{2}\right)\right) \\
& =-\frac{\tau}{2} \sum_{n=2}^{N-1} c\left(\delta u_{h}^{n}, \delta\left(u_{h}^{n+1}-u_{h}^{n}\right)\right)+\frac{\tau}{2}\left(c\left(\delta u_{h}^{N}, u_{h}^{N}\right)-c\left(\delta u_{h}^{1}, u_{h}^{2}\right)\right) \\
& =-\frac{\tau}{2} \sum_{n=2}^{N-1} c\left(\delta u_{h}^{n}, \delta \delta u_{h}^{n+1}\right)+\frac{\tau}{2}\left(c\left(\delta u_{h}^{N}, u_{h}^{N}\right)-c\left(\delta u_{h}^{1}, u_{h}^{2}\right)\right)
\end{aligned}
$$

We easily have

$$
-\frac{\tau}{2} \sum_{n=2}^{N-1} c\left(\delta u_{h}^{n}, \delta \delta u_{h}^{n+1}\right) \leq C \operatorname{Co} \sum_{n=1}^{N-1}\left\|\delta u_{h}^{n+1}-P_{0}\left(\delta u_{h}^{n+1}\right)\right\|^{2}+C \operatorname{Co} \sum_{n=1}^{N-1}\left\|\delta \delta u_{h}^{n+1}\right\|^{2} .
$$


The next term can similarly be bounded:

$$
\begin{aligned}
\frac{\tau}{2}\left(c\left(\delta u_{h}^{N}, u_{h}^{N}\right)-c\left(\delta u_{h}^{1}, u_{h}^{2}\right)\right) \leq & C \mathrm{Co}\left\|\delta u_{h}^{N}-P_{0}\left(\delta u_{h}^{N}\right)\right\|^{2}+C \operatorname{Co}\left\|u_{h}^{N}\right\|^{2} \\
& +C \operatorname{Co}\left\|\delta u_{h}^{1}-P_{0}\left(\delta u_{h}^{1}\right)\right\|^{2}+C \operatorname{Co}\left\|u_{h}^{2}\right\|^{2} .
\end{aligned}
$$

Hence, we arrive at

$$
D_{1} \leq C \operatorname{Co} \sum_{n=1}^{N-1}\left\|\delta u_{h}^{n+1}-P_{0}\left(\delta u_{h}^{n+1}\right)\right\|^{2}+C \operatorname{Co} \sum_{n=1}^{N-1}\left\|\delta \delta u_{h}^{n+1}\right\|^{2}+C \operatorname{Co}\left\|u_{h}^{N}\right\|^{2}+C \operatorname{Co}\left\|u_{h}^{N}\right\|^{2} .
$$

Which combined with the estimate for $D_{2}$ gives

$$
S_{1} \leq C \mathrm{Co} \sum_{n=1}^{N-1}\left\|\delta u_{h}^{n+1}-P_{0}\left(\delta u_{h}^{n+1}\right)\right\|^{2}+C \operatorname{Co} \sum_{n=1}^{N-1}\left\|\delta \delta u_{h}^{n+1}\right\|^{2}+C \operatorname{Co}\left\|u_{h}^{N}\right\|^{2}+C \operatorname{Co}\left\|u_{h}^{2}\right\|^{2} .
$$

Using (5.16) and combining the above inequalities we get

$$
\begin{aligned}
\frac{1}{2}\left\|u_{h}^{N}\right\|^{2}-\frac{1}{2}\left\|u_{h}^{1}\right\|^{2}+\left\|\left|\bar{u}_{h} \|\right|^{2} \leq\right. & \frac{1}{16}\left\|\bar{u}_{h}\right\|\left\|^{2}+8 \tau \sum_{n=1}^{N-1}\right\| L^{n+1}\left\|_{h}^{2}+\frac{c_{L}}{32} \tau \sum_{n=1}^{N-1}\right\| u_{h}^{n+1}\left\|^{2}+C \operatorname{Co}\right\| u_{h}^{N} \|^{2} \\
& +C \operatorname{Co}(1+\gamma) \sum_{n=1}^{N-1}\left\|\delta \delta u_{h}^{n+1}\right\|^{2}+C \operatorname{Co} \sum_{n=1}^{N-1}\left\|\delta u_{h}^{n+1}-P_{0}\left(\delta u_{h}^{n+1}\right)\right\|^{2} \\
& +C \operatorname{Co}\left\|u_{h}^{2}\right\|^{2} .
\end{aligned}
$$

Applying (5.9) we get

$$
\begin{aligned}
\frac{1}{2}\left\|u_{h}^{N}\right\|^{2}-\frac{1}{2}\left\|u_{h}^{1}\right\|^{2}+\left\|\left|\bar{u}_{h} \|\right|^{2} \leq\right. & \left(\frac{1}{16}+C \mathrm{Co}^{2}\right)\left\|\bar{u}_{h}\right\|\left\|^{2}+(8+C \mathrm{Co}) \tau \sum_{n=1}^{N-1}\right\| L^{n+1} \|_{h}^{2} \\
& +\left(\frac{c_{L}}{32}+\tau C \operatorname{Co}\|\nabla \beta\|_{\infty}^{2}\right) \tau \sum_{n=1}^{N-1}\left\|u_{h}^{n+1}\right\|^{2}+C \operatorname{Co}\left\|u_{h}^{N}\right\|^{2}+C \operatorname{Co}\left\|u_{h}^{2}\right\|^{2} .
\end{aligned}
$$

Taking Co sufficiently small we arrive at

$$
\begin{aligned}
\frac{1}{4}\left\|u_{h}^{N}\right\|^{2}+\frac{1}{2}\left\|\mid \bar{u}_{h}\right\| \|^{2} \leq & \frac{1}{2}\left\|u_{h}^{1}\right\|^{2}+9 \tau \sum_{n=1}^{N-1}\left\|L^{n+1}\right\|_{h}^{2} \\
& +\left(\frac{c_{L}}{32}+\frac{\tau}{4}\|\nabla \beta\|_{\infty}^{2}\right) \tau \sum_{n=1}^{N-1}\left\|u_{h}^{n+1}\right\|^{2}+\frac{1}{4}\left\|u_{h}^{2}\right\|^{2} .
\end{aligned}
$$

The inequality (5.12) follows from the above inequality and the discrete Gronwall's inequality (2.23).

Case 3. Pe $\geq 1, p \geq 1$. We use the same estimates for $S_{2}$ and $S_{3}$ as in Case 2 above. Then inspecting the proof of the estimate for $S_{1}$ in Case 2 we see that we could instead have shown,

$$
S_{1} \leq C \sqrt{\tau}\left(\mathrm{Co}_{4 / 3}\right)^{3 / 2} \sum_{n=1}^{N-1}\left\|\delta u_{h}^{n+1}\right\|^{2}+\epsilon \sum_{n=1}^{N-1}\left\|\delta \delta u_{h}^{n+1}\right\|^{2}+C \mathrm{Co}\left\|u_{h}^{N}\right\|^{2}+C \mathrm{Co}\left\|u_{h}^{2}\right\|^{2},
$$

where $\epsilon$ is a sufficiently small number. Now using (5.10) and (5.4) with $\max \left\{\mathrm{Co}, \mathrm{Co}_{4 / 3}\right\}$ sufficiently small we get.

$$
S_{1} \leq C \mathrm{Co}\left\|u_{h}^{N}\right\|^{2}+C \mathrm{Co}\left\|u_{h}^{2}\right\|^{2}+C \mathrm{Co}\left\|\bar{u}_{h}\right\|^{2}+9 \tau \sum_{n=1}^{N-1}\left\|L^{n+1}\right\|_{h}^{2}+\tau \sum_{n=1}^{N-1}\left\|u_{h}^{n+1}\right\|^{2} .
$$

Then we can proceed as we did in the proof of Case 2 to prove (5.12). 
TABLE 1. Table showing the Courant numbers and stabilization parameters used for the different methods.

\begin{tabular}{lll}
\hline \hline Method & Co & $\gamma$ \\
\hline BDF2/P1 & 0.15 & 0.01 \\
BDF2/P2 & 0.05 & 0.005 \\
AB2/P1 & 0.3 & 0.01 \\
AB2/P2 & 0.1 & 0.005 \\
AB3/P2 & 0.025 & 0.001 \\
AB3/P3 & 0.025 & 0.0003 \\
\hline
\end{tabular}

\section{Numerical EXAMPLES}

We consider the methods applied to the pure transport problem. More specifically, we consider two methods obtained in the limit of vanishing diffusion: the second order backward differentiation with extrapolation (BDF2) and the second order Adams-Bashforth (AB2) scheme, both of which are covered by the above analysis. For piecewise affine approximation $\left(P_{1}\right)$ we use the hyperbolic CFL, $\tau=\operatorname{Co} h\left(\|\beta\|_{\infty}=1\right)$ and for piecewise quadratic approximation we use the $4 / 3$-CFL, $\tau=\mathrm{Co} h^{4 / 3}$. Numerical experiments show that with $P_{1}$ approximation the methods are stable under hyperbolic CFL, only when $\gamma>0$ (i.e. when stabilization is present). We also observed that for $P_{2}$ the $4 / 3$-CFL is necessary for all $\gamma \geq 0$. The values of Courant numbers and stabilization parameters used for the different methods are given in Table 1 . We stress that these values are not limit values for stability for each case, but rather values that produced good results in all the experiments performed.

We also consider a numerical example using the third order Adams-Bashforth (AB3) scheme, a scheme with non-trivial imaginary stability boundary [16] that is expected to be stable under hyperbolic CFL, independent of the value of $\gamma$. This also turns out to be the case. For this method we show the results both for the stabilized and the unstabilized method to show that even though the time integrator is stable and boundary conditions are imposed weakly, strong gradients destroy the solution globally irrespective of polynomial approximation order unless stabilization is added.

For the first example we consider transport in the disc $\Omega:=\left\{(x, y) \in \mathbb{R}^{2}: x^{2}+y^{2}<1\right\}$ under the velocity field $\beta=(y,-x)$. Approximations are computed on a series of unstructured mehes with nele $=40,80,160,320$ elements along the disc perimeter. We let $f=0$ and consider two different functions $u_{0}$ as initial data. One is smooth,

$$
u_{0}=e^{-30\left((x-0.5)^{2}+y^{2}\right)}
$$

and one is rough

$$
\tilde{u}_{0}= \begin{cases}1 & \sqrt{(x+0.5)^{2}+y^{2}}<0.2 \\ 0 & \text { otherwise. }\end{cases}
$$

The velocity field simply turns the disc with the initial data and we compute one turn so that the final solution should be equal to the inital data. Two numerical experiments are considered, compute $u$ for the initial data $u_{0}$ and $u_{0}+\tilde{u}_{0}$. We compute the global error in the material derivative over the space time domain, for BDF2

$$
\left(\tau \sum_{n=2}^{N}\left\|D_{\tau} u_{h}^{n+1}+\beta \cdot \nabla \tilde{u}_{h}^{n+1}\right\|^{2}\right)^{\frac{1}{2}}, \quad \text { and for AB2, }\left(\tau \sum_{n=2}^{N}\left\|\tau^{-1} \delta u_{h}^{n+1}+\beta \cdot \nabla \hat{u}_{h}^{n+\frac{1}{2}}\right\|^{2}\right)^{\frac{1}{2}} .
$$

In all graphics the material derivative is indicated by circle markers. We also report the global $L^{2}$-norm of the error at the final time, indicated by square markers. In the case where both the rough and the smooth initial data are combined we compute the error obtained in the smooth part, i.e. the $L^{2}$-norm over $\{(x, y) \in \Omega: x>0\}$. This local error is indicated by triangle markers. 

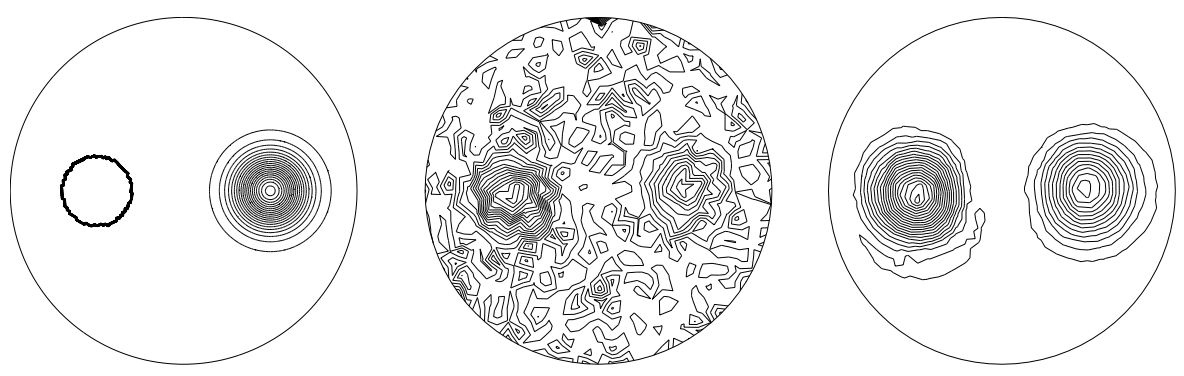

FiguRE 1. From left to right: rough initial data on fine mesh $\left(u_{0}+\tilde{u}_{0}\right)$, unstabilized solution final time solution (computed using Crank-Nicolson), stabilized final time solution (both nele $=80$, one turn).

In Figure 1 we show in the left panel the smooth and rough initial data $\left(u_{0}+\tilde{u}_{0}\right)$. In the middle panel the solution after one turn without stabilization (computed using implicit Crank-Nicolson) and in the right panel the solution after one turn with stabilization, in both cases nele $=80$. We see that the sharp layers are strongly smeared on this coarse mesh when the stabilized method is used, but contrary to the unstabilized case the smooth part of the solution is accurately captured.

In Figure 2 we compare the convergence of the BDF2 and AB2 methods with $P_{1}$ and $P_{2}$ elements for the smooth initial data. The convergence rates predicted by theory for both stabilized methods and approximation spaces are verified both for the $L^{2}$-error and in the material derivative. Both methods have very similar errors. In Figure 3 we see that in the presence of rough portions in the solution the stabilized methods still have optimal convergence in the $L^{2}$-norm in the part where the solution is smooth. We also observe that thanks to the stabilization the material derivative has only moderate growth under refinement, less than the $O\left(h^{-\frac{1}{2}}\right)$ predicted by theory. This is known not to be true for cG methods without stabilization.

\subsection{An example with inflow and outflow and weakly imposed boundary conditions}

Here we consider transport in the unit square with $\beta=(1,0)^{T}$. Structured meshes with nele $=40,80,160,320$ elements on each side are used. The initial data consists of a cylinder of radius $r=0.2$ centered in the middle of the square and a Gaussian centered on the left boundary (see Fig. 4, left plot). The exact shapes are the same as those of the previous example, (6.1) and (6.2). We compute the solution over the interval $(0,1]$ so that the cylinder leaves the domain at $t=0.7$ and at $t=1$ the Gaussian is centered at on the right boundary (see Fig. 4, right plot). Observe that from $t=0.7$ the solution is smooth. The time dependent inflow boundary condition is imposed weakly. The convergence of the $L^{2}$-error at final times for the BDF2 and AB2 approaches is shown in Figure $5(h=1 /$ nele, nele $=40,80,160,320)$. We see that for both methods the $P_{1}$ and $P_{2}$ approximations have optimal convergence to the smooth final time solution, which is known not to hold for the cG method without stabilization. This will be verified in the next section.

\subsection{Higher order time integrator: Adams-Bashforth 3}

Here we consider the same test case as in the previous section, but using the third order Adam-Bashforth scheme. In this case the scheme is similar to AB2, but the extrapolation takes the form

$$
\hat{v}^{n+1}:=\frac{23}{12} v^{n}-\frac{16}{12} v^{n-1}+\frac{5}{12} v^{n-2} .
$$

For this test case we compare the results with or without stabilization. We note that since the scheme has nontrivial imaginary stability boundary, both the stabilized and unstabilized methods are expected to be $L^{2}$-stable. This is also verified by the graphics in Figure 6. The Galerkin FEM without stabilization is distinguished by 

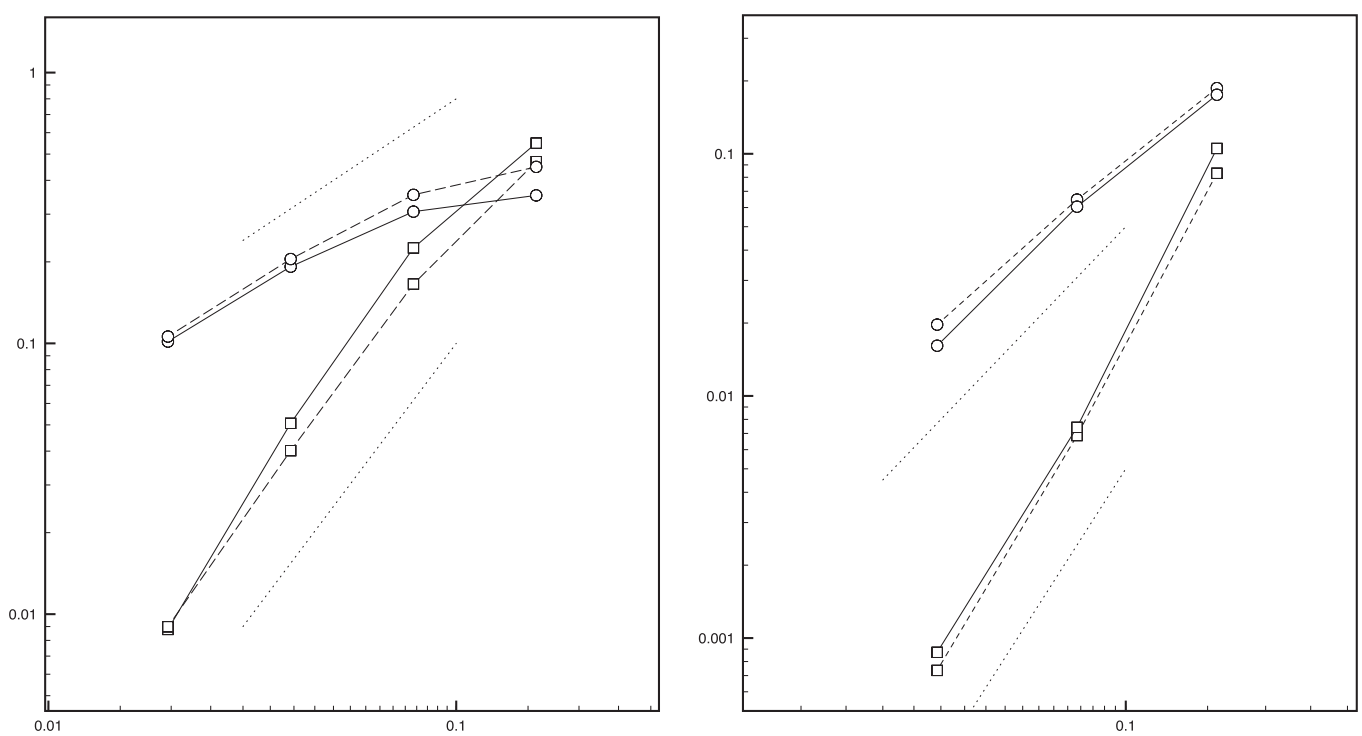

Figure 2. Comparison BDF2 (full line) and AB2 (dashed line) method with $P_{1}$ (left) and $P_{2}$ (right) approximation, with globally smooth initial data (Eq. (6.1)). The error in material derivative has circle markers. The global $L^{2}$-error has square markers. The dotted reference lines have slope 1,2 from top to bottom in the left plot and 2,3 in the right plot.
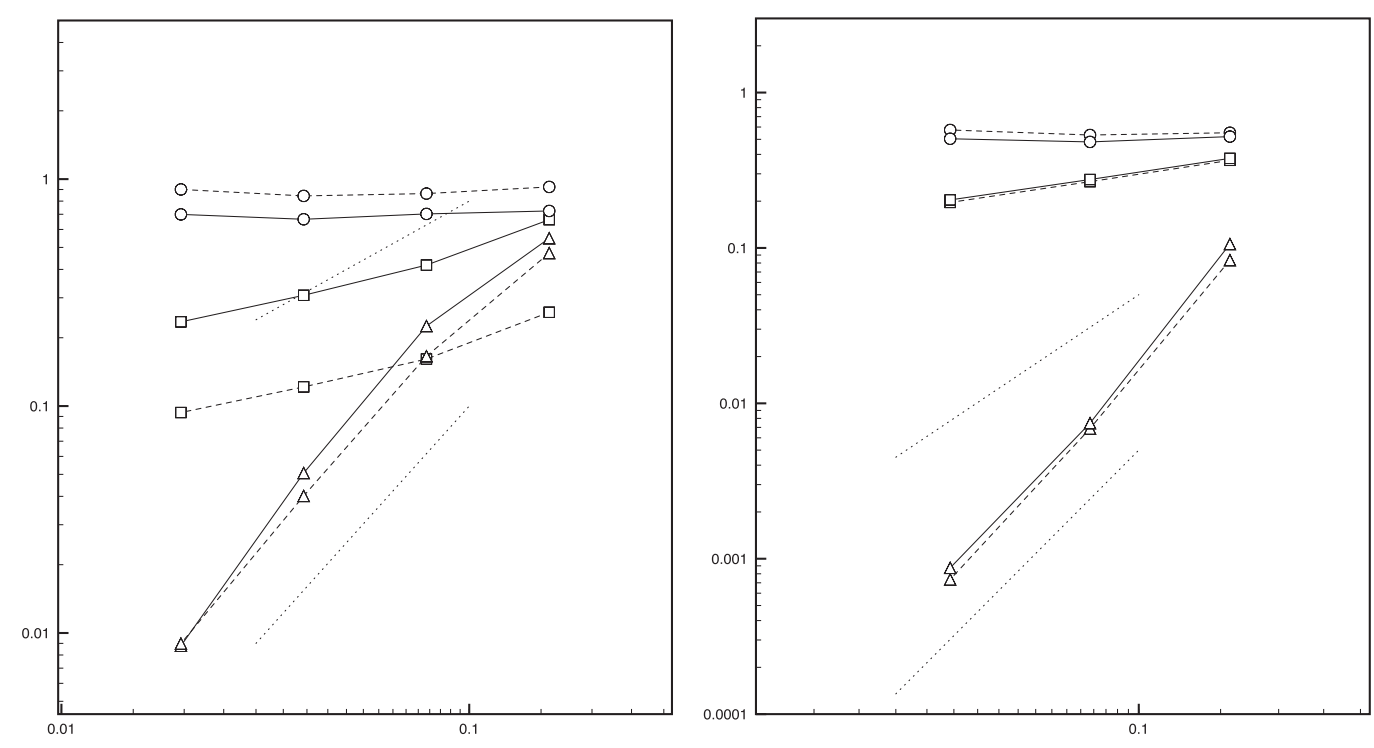

Figure 3. Comparison BDF2 (full line) and AB2 (dashed line) method with $P_{1}$ (left) and $P_{2}$ (right). Initial data from Figure 1 (left plot). The error in material derivative has circle markers. The global $L^{2}$-error has square markers and the local $L^{2}$-error has triangle markers. The dotted reference lines have slope 1,2 from top to bottom in the left plot and 2,3 in the right. 

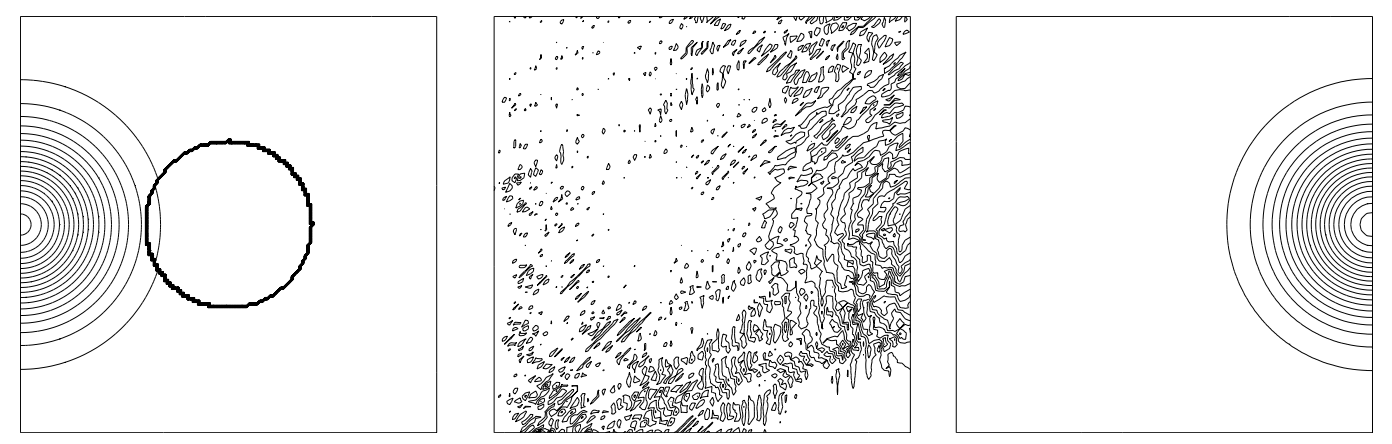

Figure 4. From left to right: initial data on fine mesh, unstabilized solution final time solution ( $P_{1}$, computed using Crank-Nicolson), stabilized final time solution (both nele $=80$, final time $t=1)$.
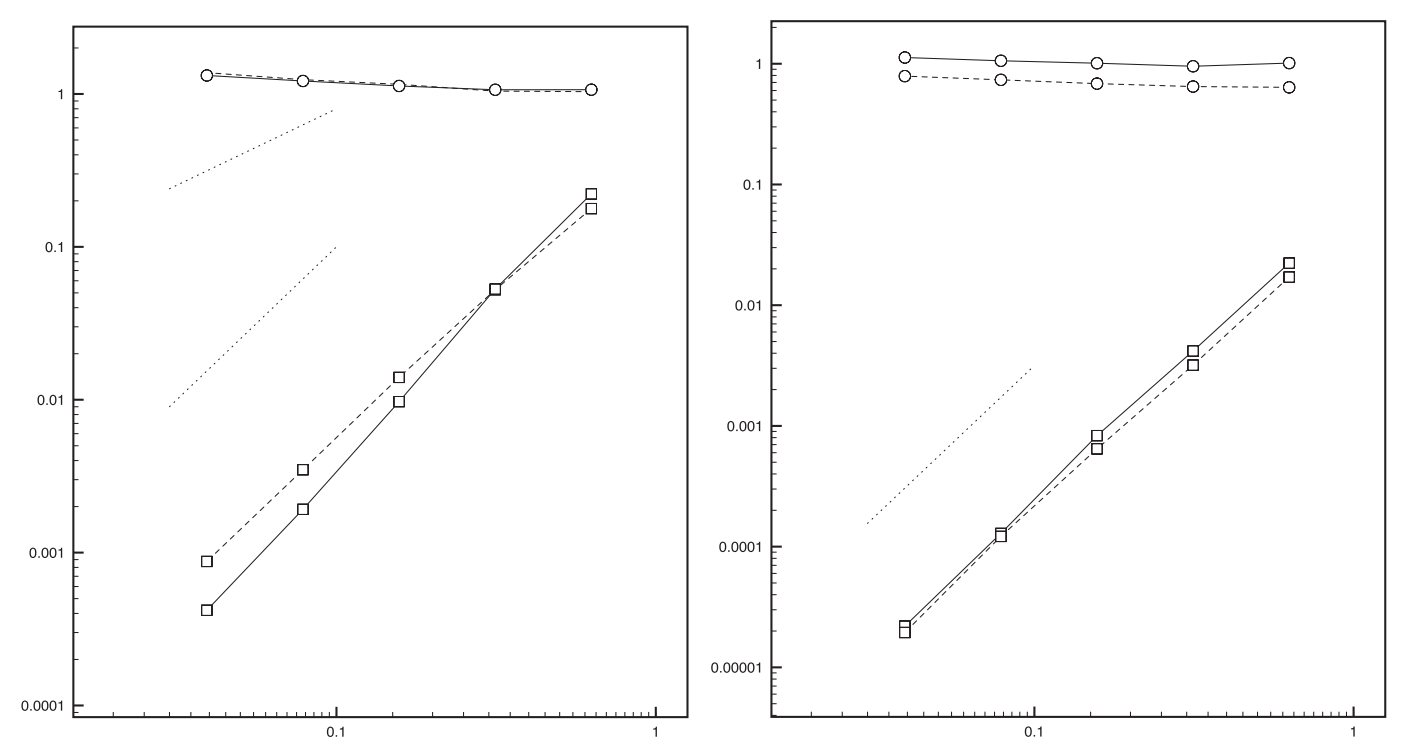

Figure 5. Comparison BDF2 (full line) and AB2 (dashed line) method with $P_{1}$ (left) and $P_{2}$ (right). Initial data from Figure 4 (left plot). The error in material derivative over the space time domain has circle markers. The final time global $L^{2}$-error has square markers. The dotted reference lines have slope 1,2 from top to bottom in the left plot and 2.5 in the right.

filled markers in the graphics. In Figure 6, left plot, we present the result for $P_{2}$ finite elements. It is clear that the solution of the stabilized method satifies the $O\left(h^{2.5}\right)$ bound predicted by theory (illustrated by the lower dotted line). Without stabilization the method has approximately $O\left(h^{\frac{1}{2}}\right)$ (upper dotted line) convergence for the smooth final time solution.

In the right plot we present the result for $P_{3}$ finite elements. Also here the stabilized method has the expected $O\left(h^{3.5}\right)$ convergence (illustrated by the lower dotted line) and the unstabilized method fails to capitalize on the increased order of the method. Its order remains at $O\left(h^{\frac{1}{2}}\right)$ (upper dotted line). As a consequence the stabilized method is more accurate by more than six orders of magnitude on the finest mesh. 

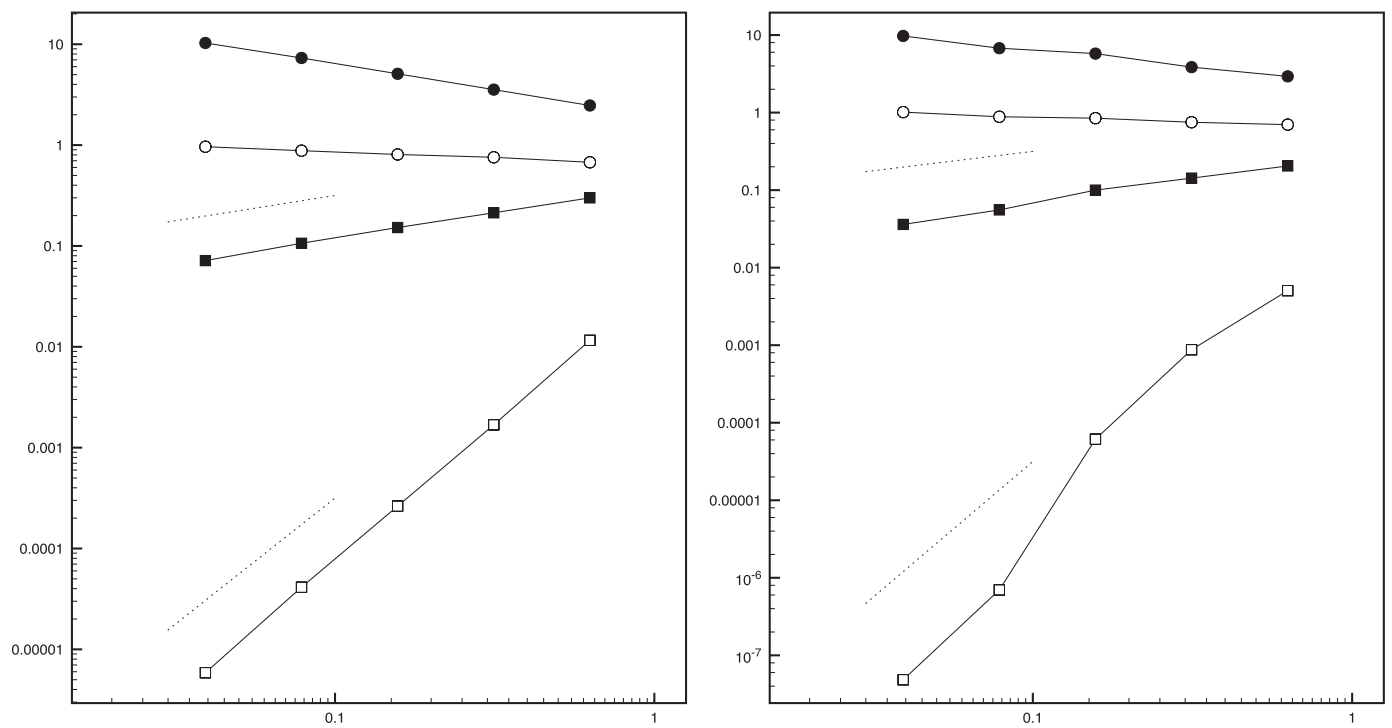

Figure 6. Comparison AB3 method with $P_{2}$ (left plot) and $P_{3}$ (right plot) polynomial approximation. Initial data from Figure 4 (left plot). The error in material derivative over the space time domain has circle markers. The global final time $L^{2}$-error has square markers. Filled markers indicate that no stabilization has been used. The upper dotted reference lines have slope 0.5 in both graphics and the lower ones have slopes 2.5 (left) and 3.5 (right).

\section{CONCLUding REMARKS}

In this paper we have considered the use of implicit-explicit time integrators together with symmetric stabilization methods. An important advantage is that the often nonlinear convection term is handled explicitly as well as the stabilization, which otherwise is known to extend the system matrix. Two second order methods were considered that are appealing in applications for their simplicity, but that have trivial imaginary stability boundary in the limit of vanishing diffusion. We prove that nevertheless these methods can be used together with stabilized FEM (or upwind discontinuous Galerkin method) under CFL conditions that allow for an optimal matching of errors in space and time. The present work opens for several interesting research venues such as the use of predictor-corrector methods [16] in combination with stabilized FEM for first order pde, or higher order IMEX-schemes based on Adams-Bashforth/Adams-Moulton combinations for singularly perturbed second order systems such as convection-diffusion or the Navier-Stokes' equations.

\section{REFERENCES}

[1] G. Akrivis, Implicit-explicit multistep methods for nonlinear parabolic equations. Math. Comp. 82 (2013) 45-68.

[2] G. Akrivis, Stability of implicit and implicit-explicit multistep methods for nonlinear parabolic equations. IMA J. Numer. Anal. 38 (2018) 1768-1796.

[3] G. Akrivis, M. Crouzeix and C. Makridakis, Implicit-explicit multistep finite element methods for nonlinear parabolic problems. Math. Comp. 67 (1998) 457-477.

[4] U.M. Ascher, S.J. Ruuth and B.T.R. Wetton, Implicit-explicit methods for time-dependent partial differential equations. SIAM J. Numer. Anal. 32 (1995) 797-823.

[5] U.M. Ascher, S.J. Ruuth and R.J. Spiteri, Implicit-explicit Runge-Kutta methods for time-dependent partial differential equations. Appl. Numer. Math. 25 (1997) 151-167. Special issue on time integration (Amsterdam, 1996).

[6] G.A. Baker, V.A. Dougalis and O.A. Karakashian, On a higher order accurate fully discrete Galerkin approximation to the Navier-Stokes equations. Math. Comp. 39 (1982) 339-375.

[7] E. Burman and A. Ern, Continuous interior penalty $h p$-finite element methods for advection and advection-diffusion equations. Math. Comp. 76 (2007) 1119-1140. 
[8] E. Burman and A. Ern, Implicit-explicit Runge-Kutta schemes and finite elements with symmetric stabilization for advectiondiffusion equations. ESAIM: M2AN 46 (2012) 681-707.

[9] E. Burman and M.A. Fernández, Finite element methods with symmetric stabilization for the transient convection-diffusionreaction equation. Comput. Methods Appl. Mech. Eng. 198 (2009) 2508-2519.

[10] E. Burman, A. Ern and M.A. Fernández, Explicit Runge-Kutta schemes and finite elements with symmetric stabilization for first-order linear PDE systems. SIAM J. Numer. Anal. 48 (2010) 2019-2042.

[11] E. Burman, A. Ern and M.A. Fernández, Fractional-step methods and finite elements with symmetric stabilization for the transient Oseen problem. ESAIM: M2AN 51 (2017) 487-507.

[12] B. Cockburn and C.-W. Shu, TVB Runge-Kutta local projection discontinuous Galerkin finite element method for conservation laws. II. General framework. Math. Comp. 52 (1989) 411-435.

[13] M. Crouzeix, Une méthode multipas implicite-explicite pour l'approximation des équations d'évolution paraboliques. Numer. Math. 35 (1980) 257-276.

[14] C. Dawson, Godunov-mixed methods for advection-diffusion equations in multidimensions. SIAM J. Numer. Anal. 30 (1993) 1315-1332.

[15] J. Douglas, Jr. and T. Dupont, Galerkin methods for parabolic equations. SIAM J. Numer. Anal. 7 (1970) 575-626.

[16] M.L. Ghrist, B. Fornberg and J.A. Reeger, Stability ordinates of Adams predictor-corrector methods. BIT 55 (2015) 733-750.

[17] W. Hundsdorfer, Trapezoidal and midpoint splittings for initial-boundary value problems. Math. Comp. 67 (1998) $1047-1062$.

[18] W. Hundsdorfer, Partially implicit BDF2 blends for convection dominated flows. SIAM J. Numer. Anal. 38 (2001) $1763-1783$.

[19] D. Levy and E. Tadmor, From semidiscrete to fully discrete: stability of Runge-Kutta schemes by the energy method. SIAM Rev. 40 (1998) 40-73.

[20] A. Mazzia, L. Bergamaschi, C.N. Dawson and M. Putti, Godunov mixed methods on triangular grids for advection-dispersion equations. Comput. Geosci. 6 (2002) 123-139.

[21] R.C. Moura, A. Cassinelli, A.F.C. da Silva, E. Burman and S.J. Sherwin, Gradient jump penalty stabilisation of spectral/hp element discretisation for under-resolved turbulence simulations. Comput. Methods Appl. Mech. Eng. 388 (2022) 114200.

[22] A.K. Pani, V. Thomée and A.S. Vasudeva Murthy, A first-order explicit-implicit splitting method for a convection-diffusion problem. Comput. Methods Appl. Math. 20 (2020) 769-782.

[23] V. Thomée, Galerkin Finite Element Methods for Parabolic Problems. Vol. 25 of Springer Series in Computational Mathematics, 2nd edition. Springer-Verlag, Berlin (2006).

[24] J.M. Varah, Stability restrictions on second order, three level finite difference schemes for parabolic equations. SIAM J. Numer. Anal. 17 (1980) 300-309.

[25] H. Wang, Y. Liu, Q. Zhang and C.-W. Shu, Local discontinuous Galerkin methods with implicit-explicit time-marching for time-dependent incompressible fluid flow. Math. Comp. 88 (2019) 91-121.

[26] H. Wang, Q. Zhang and C.-W. Shu, Implicit-explicit local discontinuous Galerkin methods with generalized alternating numerical fluxes for convection-diffusion problems. J. Sci. Comput. 81 (2019) 2080-2114.

[27] Q. Zhang and C.-W. Shu, Error estimates to smooth solutions of Runge-Kutta discontinuous Galerkin methods for scalar conservation laws. SIAM J. Numer. Anal. 42 (2004) 641-666.

[28] Q. Zhang and C.-W. Shu, Stability analysis and a priori error estimates of the third order explicit Runge-Kutta discontinuous Galerkin method for scalar conservation laws. SIAM J. Numer. Anal. 48 (2010) 1038-1063.

\section{Subscribe to Open (S2O) A fair and sustainable open access model}

This journal is currently published in open access under a Subscribe-to-Open model (S2O). S2O is a transformative model that aims to move subscription journals to open access. Open access is the free, immediate, online availability of research articles combined with the rights to use these articles fully in the digital environment. We are thankful to our subscribers and sponsors for making it possible to publish this journal in open access, free of charge for authors.

\section{Please help to maintain this journal in open access!}

Check that your library subscribes to the journal, or make a personal donation to the S2O programme, by contacting subscribers@edpsciences.org

More information, including a list of sponsors and a financial transparency report, available at: https://www. edpsciences.org/en/maths-s2o-programme 